\title{
Zero-point energy of ultracold atoms
}

\author{
Luca Salasnich ${ }^{1,2}$ and Flavio Toigo ${ }^{1}$ \\ ${ }^{1}$ Dipartimento di Fisica e Astronomia "Galileo Galilei" and CNISM, \\ Università di Padova, via Marzolo 8, 35131 Padova, Italy \\ ${ }^{2}$ CNR-INO, via Nello Carrara, 1 - 50019 Sesto Fiorentino, Italy
}

\begin{abstract}
We analyze the divergent zero-point energy of a dilute and ultracold gas of atoms in $D$ spatial dimensions. For bosonic atoms we explicitly show how to regularize this divergent contribution, which appears in the Gaussian fluctuations of the functional integration, by using three different regularization approaches: dimensional regularization, momentum-cutoff regularization and convergence-factor regularization. In the case of the ideal Bose gas the divergent zero-point fluctuations are completely removed, while in the case of the interacting Bose gas these zero-point fluctuations give rise to a finite correction to the equation of state. The final convergent equation of state is independent of the regularization procedure but depends on the dimensionality of the system and the two-dimensional case is highly nontrivial. We also discuss very recent theoretical results on the divergent zero-point energy of the $D$-dimensional superfluid Fermi gas in the BCS$\mathrm{BEC}$ crossover. In this case the zero-point energy is due to both fermionic single-particle excitations and bosonic collective excitations, and its regularization gives remarkable analytical results in the BEC regime of composite bosons. We compare the beyond-mean-field equations of state of both bosons and fermions with relevant experimental data on dilute and ultracold atoms quantitatively confirming the contribution of zero-point-energy quantum fluctuations to the thermodynamics of ultracold atoms at very low temperatures.
\end{abstract}

\section{Contents}

1 Introduction

2 Functional integration for bosonic superfluids 2.1 Ideal Bose gas . . . . . . . . . . . . . . . 6 6

Preprint submitted to Physics Reports November 5, 2018 
2.2 Interacting Bose gas . . . . . . . . . . . . . . 9

3 Regularization $\quad 13$

3.1 Regularization and scattering theory . . . . . . . . . . 13

3.2 Dimensional regularization $(\mathrm{DR}) \ldots \ldots \ldots \ldots . \ldots . \ldots$

3.2 .1 Ideal Bose gas - DR . . . . . . . . . . . . 15

3.2.2 Repulsive Bose gas in 1D and 3D - DR . . . . . . 16

3.2.3 Repulsive Bose gas in 2D - DR . . . . . . . . . . . 17

3.3 Momentum-cutoff regularization (MCR) . . . . . . . . 18

3.3.1 Ideal Bose gas - MCR . . . . . . . . . . . . . . . 19

3.3.2 Repulsive Bose gas in 1D and 3D - MCR . . . . . . 19

3.3.3 Repulsive Bose gas in 2D - MCR . . . . . . . . . 21

3.4 Convergence-factor regularization $(\mathrm{CFR}) \ldots \ldots \ldots . . \ldots 23$

3.4 .1 Ideal Bose gas - CFR . . . . . . . . . . . . 23

3.4.2 Repulsive Bose gas in 1D and 3D - CFR . . . . . . . 23

3.4.3 Repulsive Bose gas in 2D - CFR . . . . . . . . . 24

4 Experiments vs theory for bosonic superfluids 25

4.1 Repulsive Bose gas in 3D . . . . . . . . . . . . 25

4.2 Repulsive Bose gas in 2D . . . . . . . . . . . . . . 27

4.3 Repulsive Bose gas in 1D . . . . . . . . . . . . 28

5 Functional integration for fermionic superfluids 29

5.1 Three-dimensional attractive Fermi gas . . . . . . . . . 32

5.2 Two-dimensional attractive Fermi gas . . . . . . . . . 36

5.3 One-dimensional attractive Fermi gas . . . . . . . . . . . . 39

6 Experiments vs theory for fermionic superfluids 41

6.1 Attractive Fermi gas in 3D . . . . . . . . . . 41

6.2 Attractive Fermi gas in $2 \mathrm{D} \ldots \ldots \ldots \ldots$

6.3 Attractive Fermi gas in $1 \mathrm{D} \ldots \ldots \ldots$

7 Conclusions 46

\section{Introduction}

The experimental achievement of Bose-Einstein condensation (Bose, 1924; Einstein, 1924) with dilute and ultracold alkali-metal atoms (Anderson et al., 1995; Bradley et al., 1995; Davis et al., 1995) has triggered many theoretical investigations and comprehensive reviews (Shi and Griffin, 1998; Dalfovo et al., 1999; Leggett, 2001; Andersen, 2004) on the properties of the weakly-interacting 
Bose gas. Experiments with ultracold and dilute atomic gases in quasi-1D (Paredes et al., 2004; Kinoshita et al., 2004) and quasi-2D configurations (Hadzibabic et al., 2006; Hung et al., 2011; Makhalov et al., 2014) have renewed the interest on the properties of Bose gases with reduced dimensionality, where quantum fluctuations play a relevant role (Mermin and Wagner, 1966; Hohenberg, 1967; Coleman, 1973).

The study of the uniform weakly-interacting Bose gas in 1, 2 and 3 dimensions has a long history. In their seminal papers Bogoliubov (1947), Lee and Yang (1957) and Lee, Huang and Yang (1957) investigated the properties of a Bose gas with hard-core repulsion in three dimensions: Bogoliubov calculated the zero-temperature quantum depletion, while Lee and Yang and Lee, Huang and Yang evaluated the leading quantum corrections to the mean-field equation by properly treating the divergencies introduced by a naif treatment of the repulsion as a contact interaction. In one dimension, based on a previous investigation of the 1D Bose-Fermi mapping by Girardeau (1960), Lieb and Liniger (1963) obtained the exact equation of state of a Bose gas with contact repulsive interaction exploiting the Bethe ansatz. In the case of two spatial dimensions, Schick (1971) found that the equation of state of a uniform 2D repulsive Bose gas contains a nontrivial logarithmic term. This remarkable result was improved by Popov (1972) who obtained an equation of state which, at the leading order, reduces to Schick's one in the dilute limit. A key ingredient in the theoretical analysis of both Schick (1971) and Popov (1972) is the logarithmic behavior of the T-matrix that describes the scattering between bosons.

In this article we show that the finite-temperature equation of state of interacting bosons can be derived by functional integration (Nagaosa, 1999) of a $D$-dimensional model with a purely local potential after proper regularization of the divergence of the zero-point energy of Gaussian fluctuations. We consider three different regularization approaches: the modern dimensional regularization ('t Hooft and Veltman, 1972) used mainly in highenergy physics, the old momentum-cutoff regularization (Feynman, 1948; Pauli and Villars, 1949), still adopted in many physical contexts, and the convergence-factor renormalization used mainly in condensed-matter physics (Nagaosa, 1999; Stoof et al., 2009; Altland and Simons, 2010). We find that the divergence of the zero-point energy in an ideal Bose gas is completely removed by regularization. On the contrary, in the case of an interacting Bose gas, the zero-point energy contributes a finite correction to the equation of state even after regularization. The final equation of state is independent of the regularization procedure but depends on the dimensionality of the system. Following Braaten and Nieto (1997), Andersen (2004) and Schakel 
(2008) in the one and three-dimensional cases respectively, we recover the familiar results of Lieb and Liniger (1963) (in the quasi-condensate regime) and Lee, Huang and Yang (1957), without making explicit use of scattering theory. In the highly non-trivial two-dimensional case, we obtain exactly Popov's equation of state (Popov, 1972) through novel treatments of either dimensional or cutoff regularizations. In particular, the dimensional regularization around $D=2$ is based on a renormalization-group analysis (Schakel, 1999; Andersen and Haugerud, 2002; Chien et al., 2014) with a specific choice of the Landau pole recently used in the study of composite bosons in the 2D BCS-BEC crossover (Salasnich and Toigo, 2015). The momentum-cutoff and convergence-factor regularizations in two dimensions are based on new approaches developed for this paper. Also in the twodimensional case, both dimensional and momentum-cutoff regularizations do not require the use of T-matrix scattering theory, which is instead a key ingredient in the original derivation of Popov (1972), which is equivalent to the convergence-factor regularization. Not pretending to give a complete experimental overview, which is far beyond the scope of this paper, we analyze relevant experiments with ultracold and dilute atomic gases in 3D (Papp et al. (2008); Wild et al. (2012)) and 2D (Nascimbene et al. (2010); Yefsah et al. (2011)) which put in evidence effects of zero-point energy on the equation of state of repulsive bosons. Experiments on 1D bosons (Kinoshita et al. (2004); Paredes et al. (2004)) show that the Lieb-Liniger theory is needed to accurately describe the strong-coupling (i.e. low 1D density) regime.

In this paper we also discuss current theoretical investigations related to the regularization of the zero-point energy of a more complex physical system: the $D$-dimensional Fermi superfluid in the BCS-BEC crossover, i.e. the crossover of a fermionic superfluid from weakly-bound BCS-like Cooper pairs to the Bose-Einstein condensation (BEC) of strongly-bound molecules (Greiner et al., 2003; Chin et al., 2004; Makhalov et al., 2014). For this system there are two kinds of elementary excitations (fermionic single-particle excitations and bosonic collective excitations) which contribute to the zeropoint energy of Gaussian quantum fluctuations. Very recently we have obtained remarkable results for $D=3$ (Salasnich and Bighin, 2015) and $D=2$ (Salasnich and Toigo, 2015) removing all the divergences in the BEC regime of the crossover. Also for attractive fermions we analyze only experiments with ultracold and dilute atomic gases which display zero-temperature beyond-mean-field effects on the equation of state. The main conclusion is that 3D (Altmever et al. (2007); Levronas and Combescot (2007)) and 2D (Makhalov et al. (2014); Luick (2014); Boetcher et al. (2016)) experimental 
data are in quite good agreement with the theory when Gaussian fluctuations are taken into account. For 1D superfluid fermions we show that Gaussian fluctuations improve the mean-field theory but do not give the correct equation of state in the Tonks-like regime of impenetrable bosons (Girardeau, 1960; Gaudin, 1967), required to reproduce the observed density profiles of 1D trapped atoms (Liao et al. (2010)). General reviews of experiments with ultracold bosonic and fermionic atoms in reduced dimensions, achieved by using very anisotropic trapping potentials, can be found, for example, in Bloch, Dalibard, and Zwerger (2008) and Hadzibabic and Dalibard (2011).

\section{Functional integration for bosonic superfluids}

We consider a D-dimensional $(D=1,2,3)$ Bose gas of ultracold and dilute neutral atoms either noninteracting or with a repulsive contact interaction. We adopt the path integral formalism, where the atomic bosons are described by the complex field $\psi(\mathbf{r}, \tau)$ (Nagaosa, 1999). The Euclidean Lagrangian density of the free system in a D-dimensional box of volume $L^{D}$ and with chemical potential $\mu$ is given by

$$
\mathscr{L}=\bar{\psi}\left[\hbar \partial_{\tau}-\frac{\hbar^{2}}{2 m} \nabla^{2}-\mu\right] \psi+\frac{1}{2} g|\psi|^{4},
$$

where $g>0$ is the strength of the contact inter-atomic coupling (Nagaosa, 1999). The partition function $\mathcal{Z}$ of the system at temperature $T$ can then be written as

$$
\mathcal{Z}=\int \mathcal{D}[\psi, \bar{\psi}] \exp \left\{-\frac{S[\psi, \bar{\psi}]}{\hbar}\right\}
$$

where

$$
S[\psi, \bar{\psi}]=\int_{0}^{\hbar \beta} d \tau \int_{L^{D}} d^{D} \mathbf{r} \mathscr{L}(\psi, \bar{\psi})
$$

is the Euclidean action and $\beta \equiv 1 /\left(k_{B} T\right)$ with $k_{B}$ the Boltzmann's constant. The grand potential $\Omega$ of the system, which is a function of the thermodynamic variables $\mu, T$ and of the parameter $g$, is then obtained as

$$
\Omega=-\frac{1}{\beta} \ln \mathcal{Z} .
$$

We work in the superfluid phase where the global U(1) gauge symmetry of the system is spontaneously broken (Nagaosa, 1999). For this reason we set

$$
\psi(\mathbf{r}, \tau)=\psi_{0}+\eta(\mathbf{r}, \tau)
$$


where $\eta(\mathbf{r}, \tau)$ is the complex field of bosonic fluctuations around the order parameter $\psi_{0}$ (condensate in $3 \mathrm{D}$ or quasi-condensate in $1 \mathrm{D}$ and $2 \mathrm{D}$ ) of the system. We suppose that $\psi_{0}$ is constant in time, uniform in space and real.

\subsection{Ideal Bose gas}

First we analyze the case with $g=0$, where exact analytical results can be obtained in any spatial dimension $D$. In fact, the Euclidean action of the ideal Bose gas can be written in a diagonal form as

$$
\begin{aligned}
S[\psi, \bar{\psi}] & =-\mu \psi_{0}^{2} \hbar \beta L^{D} \\
& +\frac{1}{2} \sum_{Q}(\bar{\psi}(Q), \psi(-Q)) \mathbf{M}(Q)\left(\begin{array}{c}
\psi(Q) \\
\bar{\psi}(-Q)
\end{array}\right)
\end{aligned}
$$

where $Q=\left(\mathbf{q}, i \omega_{n}\right)$ is the $D+1$ vector denoting the momenta $\mathbf{q}$ and bosonic Matsubara frequencies $\omega_{n}=2 \pi n /(\beta \hbar)$, and

$$
\mathbf{M}(Q)=\beta\left(\begin{array}{cc}
-i \hbar \omega_{n}+\frac{\hbar^{2} q^{2}}{2 m}-\mu & 0 \\
0 & i \hbar \omega_{n}+\frac{\hbar^{2} q^{2}}{2 m}-\mu
\end{array}\right)
$$

is the diagonal inverse fluctuation propagator of the quadratic action.

Integrating over the bosonic fields $\eta(Q)$ and $\bar{\eta}(Q)$ in Eq. (6) one finds the grand potential

$$
\begin{aligned}
\Omega & =-\mu \psi_{0}^{2} L^{D}+\frac{1}{2 \beta} \sum_{Q} \ln \operatorname{Det}(\mathbf{M}(Q)) \\
& =-\mu \psi_{0}^{2} L^{D}+\frac{1}{2 \beta} \sum_{\mathbf{q}} \sum_{n=-\infty}^{+\infty} \ln \left[\beta^{2}\left(\hbar^{2} \omega_{n}^{2}+\xi_{q}^{2}\right)\right],
\end{aligned}
$$

where $\xi_{q}$ is the shifted free-particle spectrum, i.e.

$$
\xi_{q}=\frac{\hbar^{2} q^{2}}{2 m}-\mu
$$

The sum over bosonic Matsubara frequencies gives (Andersen, 2004; Kapusta, 1993; Le Bellac, 1996)

$$
\frac{1}{2 \beta} \sum_{n=-\infty}^{+\infty} \ln \left[\beta^{2}\left(\hbar^{2} \omega_{n}^{2}+\xi_{q}^{2}\right)\right]=\frac{\xi_{q}}{2}+\frac{1}{\beta} \ln \left(1-e^{-\beta \xi_{q}}\right) .
$$


Strictly speaking, there should also be an additional infinite term on the right side of Eq. (10). However, since this infinite constant is independent of $\beta$ and $\mu$, it can be neglected (Kapusta, 1993; Le Bellac, 1996). The grand potential finally reads

$$
\Omega=\Omega_{0}+\Omega^{(0)}+\Omega^{(T)},
$$

where

$$
\Omega_{0}=-\mu \psi_{0}^{2} L^{D}
$$

is the grand potential of the order parameter,

$$
\Omega^{(0)}=\frac{1}{2} \sum_{\mathbf{q}} \xi_{q}
$$

is the zero-point energy of bosonic single-particle excitations, i.e. the zerotemperature contribution of quantum fluctuations, and

$$
\Omega^{(T)}=\frac{1}{\beta} \sum_{\mathbf{q}} \ln \left(1-e^{-\beta \xi_{q}}\right)
$$

takes into account thermal fluctuations. In the continuum limit, where $\sum_{\mathbf{q}} \rightarrow L^{D} \int d^{D} \mathbf{q} /(2 \pi)^{D}$, the zero-point energy

$$
\frac{\Omega^{(0)}}{L^{D}}=\frac{1}{2} \frac{S_{D}}{(2 \pi)^{D}} \int_{0}^{+\infty} d q q^{D-1}\left(\frac{\hbar^{2} q^{2}}{2 m}-\mu\right)
$$

of the ideal Bose gas is clearly ultraviolet divergent at any integer dimension $D$, i.e for $D=1,2,3$. Here $S_{D}=2 \pi^{D / 2} / \Gamma(D / 2)$ is the solid angle in $D$ dimensions with $\Gamma(x)$ the Euler gamma function. We shall show that this divergent zero-point energy of the ideal Bose gas is completely eliminated by dimensional regularization, or momentum-cutoff regularization, or convergence-factor regularization. Consequently, the exact grand potential of the ideal Bose gas is given by

$$
\frac{\Omega}{L^{D}}=-\mu \psi_{0}^{2}+\frac{1}{\beta L^{D}} \sum_{\mathbf{q}} \ln \left(1-e^{-\beta \xi_{q}}\right) .
$$

We notice that $\psi_{0}$ is not a free parameter but must be determined by minimizing $\Omega_{0}$, namely

$$
\left(\frac{\partial \Omega_{0}}{\partial \psi_{0}}\right)_{\mu, T, L^{D}}=0
$$


from which one finds that

$$
\psi_{0}= \begin{cases}0 & \text { if } \mu<0 \\ \text { any value } & \text { if } \mu=0\end{cases}
$$

The number density $n=N / L^{D}$ is obtained from the thermodynamic relation

$$
n=-\frac{1}{L^{D}}\left(\frac{\partial \Omega}{\partial \mu}\right)_{T, L^{D}}
$$

which gives:

$$
n=\psi_{0}^{2}+\frac{1}{L^{D}} \sum_{\mathbf{q}} \frac{1}{e^{\beta \xi_{q}}-1} .
$$

We stress that, for the ideal Bose gas, only after fixing the total density $n$ one can find the value of $\psi_{0}$ as a function of the chemical potential $\mu$ and temperature $T$. Moreover, in the continuum limit where $\sum_{\mathbf{q}} \rightarrow L^{D} \int d^{D} \mathbf{q} /(2 \pi)^{D}$, by setting $\psi_{0}=\mu=0$ from Eq. (20) one gets

$$
n=\int \frac{d^{D} \mathbf{q}}{(2 \pi)^{D}} \frac{1}{e^{\frac{\hbar^{2} q^{2}}{2 m k T^{T}}}-1}
$$

as the implicit equation determining the critical temperature $T_{c}$ for BoseEinstein condensation. It is well known (Huang, 1987) that one finds

$$
k_{B} T_{c}= \begin{cases}\text { no solution } & \text { for } D=1 \\ 0 & \text { for } D=2 \\ \frac{1}{2 \pi \zeta(3 / 2)^{2 / 3}} \frac{\hbar^{2}}{m} n^{2 / 3} & \text { for } D=3\end{cases}
$$

where $\zeta(x)$ is the Riemann zeta function. It is important to stress that, also in the absence of true Bose-Einstein condensation (as in $D=1$ and in $D=2$ for $T>0$ ), one can have quasi-condensation, i.e. algebraic-long-range-order of the two-body density matrix, where the use of the order parameter $\psi_{0}$ is still meaningful (Stoof, 2009). 


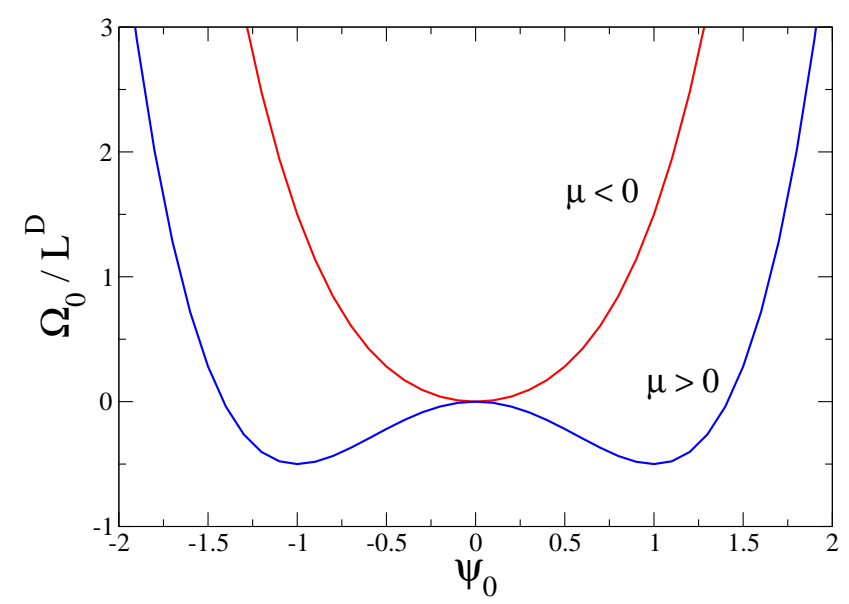

Figure 1: Mean-field grand potential $\Omega_{0}$ as a function of the real order parameter $\psi_{0}$ for an interacting Bose gas, see Eq. (24). For a positive chemical potential $\mu$ there is spontaneous symmetry breaking and the system becomes superfluid. $\Omega$ is in units of $|\mu|$ and we choose $g /|\mu|=1$. For $\mu<0$ there is single-well potential with the minimum at $\psi_{0}=0$, while for $\mu>0$ there is a double-well potential with minima at $\psi_{0} \neq 0$.

\subsection{Interacting Bose gas}

Let us now consider a system of bosons with a repulsive contact interaction, i.e. let us set $g>0$ in Eq. (11). In this case one finds immediately the partition function of the order parameter

$$
\mathcal{Z}_{0}=\exp \left\{-\frac{S_{0}}{\hbar}\right\}=\exp \left\{-\beta \Omega_{0}\right\}
$$

where the grand potential $\Omega_{0}$ reads (see Fig. 1)

$$
\frac{\Omega_{0}}{L^{D}}=-\mu \psi_{0}^{2}+\frac{1}{2} g \psi_{0}^{4} .
$$

Again, the constant, uniform and real order parameter $\psi_{0}$ is obtained by minimizing $\Omega_{0}$ as

$$
\left(\frac{\partial \Omega_{0}}{\partial \psi_{0}}\right)_{\mu, T, L^{D}}=0,
$$

from which one finds the relation between order parameter and chemical potential

$$
\mu=g \psi_{0}^{2}
$$

showing that in the superfluid broken phase the chemical potential is positive and

$$
\psi_{0}=\sqrt{\frac{\mu}{g}} .
$$


Inserting this relation into Eq. (24) we find

$$
\frac{\Omega_{0}}{L^{D}}=-\frac{\mu^{2}}{2 g} .
$$

Clearly, this equation of state is lacking important informations encoded in quantum and thermal fluctuations.

As previously pointed out, the main goal of this paper is to discuss and take into account these fluctuations, and in particular zero-temperature quantum fluctuations which are crucial in reduced dimensionalities (Mermin and Wagner, 1966; Hohenberg, 1967; Coleman, 1973). To this end we allow $\eta(\mathbf{r}, \tau) \neq 0$ in Eq. (5) and expand the action $S[\psi, \bar{\psi}]$ of Eq. (3) around $\psi_{0}$ up to quadratic (Gaussian) order in $\eta(\mathbf{r}, \tau)$ and $\bar{\eta}(\mathbf{r}, \tau)$. One finds

$$
Z=Z_{0} \int \mathcal{D}[\eta, \bar{\eta}] \exp \left\{-\frac{S_{g}[\eta, \bar{\eta}]}{\hbar}\right\}
$$

where

$$
S_{g}[\eta, \bar{\eta}]=\frac{1}{2} \sum_{Q}(\bar{\eta}(Q), \eta(-Q)) \mathbf{M}(Q)\left(\begin{array}{c}
\eta(Q) \\
\bar{\eta}(-Q)
\end{array}\right)
$$

is the Gaussian action of fluctuations in reciprocal space with $Q=\left(\mathbf{q}, i \omega_{n}\right)$ a $D+1$ vector denoting momenta $\mathbf{q}$ and bosonic Matsubara frequencies $\omega_{n}=2 \pi n /(\beta \hbar)$, and again

$$
\mathbf{M}(Q)=\beta\left(\begin{array}{cc}
-i \hbar \omega_{n}+\frac{\hbar^{2} q^{2}}{2 m}-\mu+2 g \psi_{0}^{2} & g \psi_{0}^{2} \\
g \psi_{0}^{2} & i \hbar \omega_{n}+\frac{\hbar^{2} q^{2}}{2 m}-\mu+2 g \psi_{0}^{2}
\end{array}\right)
$$

is the inverse fluctuation propagator.

Integrating over the bosonic fields $\eta(Q)$ and $\bar{\eta}(Q)$ in Eq. (29) one finds the Gaussian grand potential

$$
\begin{aligned}
\Omega_{g} & =\frac{1}{2 \beta} \sum_{Q} \ln \operatorname{Det}(\mathbf{M}(Q)) \\
& =\frac{1}{2 \beta} \sum_{\mathbf{q}} \sum_{n=-\infty}^{+\infty} \ln \left[\beta^{2}\left(\hbar^{2} \omega_{n}^{2}+E_{q}^{2}\right)\right],
\end{aligned}
$$

where

$$
E_{q}=\sqrt{\left(\frac{\hbar^{2} q^{2}}{2 m}-\mu+2 g \psi_{0}^{2}\right)^{2}-g^{2} \psi_{0}^{4}}
$$


is the familiar Bogoliubov spectrum when Eq. (26) is used. Taking into account Eq. (10), the sum over bosonic Matsubara frequencies gives (Andersen, 2004; Kapusta, 1993; Le Bellac, 1996)

$$
\frac{1}{2 \beta} \sum_{n=-\infty}^{+\infty} \ln \left[\beta^{2}\left(\hbar^{2} \omega_{n}^{2}+E_{q}^{2}\right)\right]=\frac{E_{q}}{2}+\frac{1}{\beta} \ln \left(1-e^{-\beta E_{q}}\right),
$$

and the total grand potential may then be written as

$$
\Omega=\Omega_{0}+\Omega_{g}^{(0)}+\Omega_{g}^{(T)},
$$

where $\Omega_{0}$ is given by Eq. (28) .

$$
\Omega_{g}^{(0)}=\frac{1}{2} \sum_{\mathbf{q}} E_{q}
$$

again is the zero-point energy of bosonic collective excitations, i.e. the zerotemperature contribution of quantum Gaussian fluctuations, while

$$
\Omega_{g}^{(T)}=\frac{1}{\beta} \sum_{\mathbf{q}} \ln \left(1-e^{-\beta E_{q}}\right)
$$

takes into account thermal Gaussian fluctuations. Note that if $g=0$ one finds $E_{q}=\xi_{q}$ and Eq. (14) is recovered.

We remark again that using Eq. (26) to remove the dependence on $\psi_{0}$ in $E_{q}$ one obtains the usual form for the Bogoliubov spectrum, i.e.

$$
E_{q}=\sqrt{\frac{\hbar^{2} q^{2}}{2 m}\left(\frac{\hbar^{2} q^{2}}{2 m}+2 \mu\right)} .
$$

We notice that the continuum limit of the zero-point energy for the interacting Bose gas

$$
\frac{\Omega_{g}^{(0)}}{L^{D}}=\frac{1}{2} \frac{S_{D}}{(2 \pi)^{D}} \int_{0}^{+\infty} d q q^{D-1} \sqrt{\frac{\hbar^{2} q^{2}}{2 m}\left(\frac{\hbar^{2} q^{2}}{2 m}+2 \mu\right)}
$$

is ultraviolet divergent at any integer dimension $D$.

We stress the formal similarities between Eq. (15) for the ideal Bose gas and Eq. (39) for the interacting Bose gas. In Sections 3 and 4 we shall show that the divergent zero-point energy of the interacting Bose gas, Eq. (39), gives rise to a finite correction to the equation of state, which is independent of the regularization procedure. 


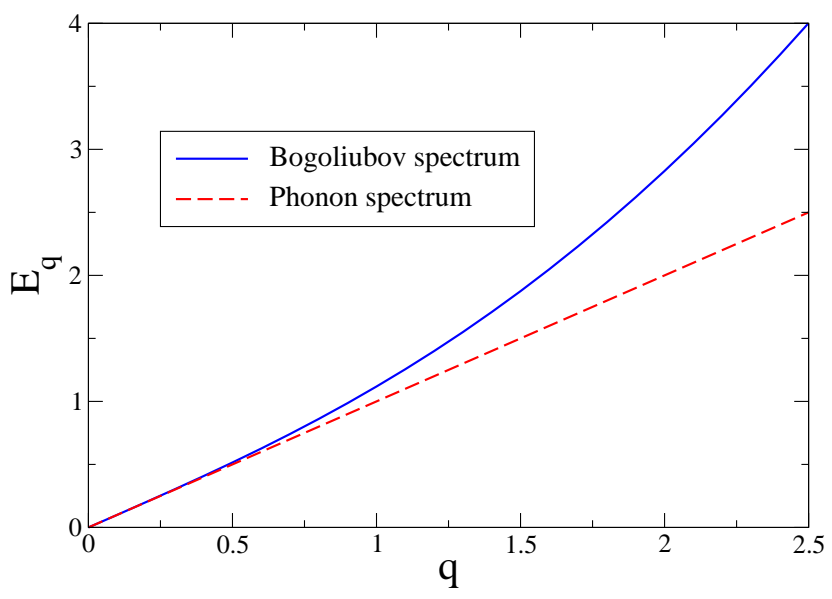

Figure 2: Bogoliubov spectrum, given by Eq. (38), and its low-momentum phonon spectrum $E_{q}=c_{B} \hbar q$, where $c_{B}=\sqrt{\mu / m}$ is the sound velocity. Energy $E_{q}$ in units of $\mu$ and momentum $q$ in units of $\sqrt{m \mu / \hbar^{2}}$.

Before concluding this section we wish to comment on two points: a) the limits of validity of the Gaussian approximation, i.e. of Bogoliubov approximation, and b) the relation between Bogoliubov's spectrum and the speed of sound.

Regarding point a), we observe that the Gaussian approximation may be seen as an expansion in terms of the adimensional parameter $\gamma=\left(m / \hbar^{2}\right) g n^{(D-2) / D}$ with $n$ the number density of bosons. So, we expect Bogoliubov's approximation to be asymptotically correct when $\gamma \ll 1$.

As for point b) we notice that in the low-momenta regime, when $\hbar q \ll$ $\sqrt{2 m \mu}$, Bogoliubov's spectrum of Eq. (39) reduces to the familiar linear phonon spectrum $E_{q}=\hbar c_{B} q$ (see Fig. 2). We stress that in general $c_{B}$ differs from the speed of sound $c_{s}$ derived from thermodynamics according to

$$
m c_{s}^{2}=\frac{\left(\frac{\partial \Omega}{\partial \mu}\right)_{T, L^{D}}}{\left(\frac{\partial^{2} \Omega}{\partial \mu^{2}}\right)_{T, L^{D}}} .
$$

Indeed, even at $T=0, c_{s}$ is a complicate function of $\mu$ and therefore of the density $n$, since the derivatives of the grand potential have contributions both from $\Omega_{0}$ and from $\Omega_{g}^{(0)}$. Only for densities such that the latter is negligible one finds $c_{s}=c_{B}$. 


\section{Regularization}

The choice of a contact interaction has allowed us to perform analytical derivations, at the price, however of obtaining divergent results.

To get finite physical values, we must therefore regularize the otherwise divergent Eq. (15) for the ideal Bose gas and Eq. (39) for the interacting Bose gas by properly renormalizing the coupling constant $g$ on the basis of some physical constant characterizing the two body scattering, such as the scattering length.

\subsection{Regularization and scattering theory}

We recall that in 3D Bogoliubov (1947) was able to remove divergent zero-point fluctuations by using canonical quantization and a regularization procedure relating the physical s-wave scattering length $a_{B}$ of the actual interatomic potential to the strength $g$ of the model contact interaction:

$$
\frac{m}{4 \pi \hbar^{2} a_{B}}=\frac{1}{g}+\frac{1}{L^{3}} \sum_{\mathbf{q}} \frac{m}{\hbar^{2} q^{2}} .
$$

This equation may be easily deduced from the Lippman-Schwinger equation for the $T$-matrix $\hat{T}(E)$ (see Stoof et al. (2009)). In the low energy limit, the Lippman-Schwinger equation renormalizes the coupling constant of a contact interaction $V(\mathbf{r})=g \delta(\mathbf{r})$ as

$$
\frac{1}{g_{r}(E)} \equiv \frac{1}{T\left(E+i 0^{+}\right)}=\frac{1}{g}+\frac{1}{L^{D}} \sum_{\mathbf{q}} \frac{m}{\hbar^{2} q^{2}-E-i 0^{+}},
$$

where $T(E)=T_{\mathbf{k}, \mathbf{k}^{\prime}}(E) \equiv\left\langle\mathbf{k}|\hat{T}(E)| \mathbf{k}^{\prime}\right\rangle$ when $k=k^{\prime}$ and $E=\hbar^{2} k^{2} / m$.

At very low energy one may relate the s-wave scattering length $a_{B}$, a physical measurable quantity, to $T(E)$ according to

$$
T\left(E+i 0^{+}\right)= \begin{cases}\frac{4 \pi \hbar^{2}}{m} \frac{a_{B}}{1+i a_{B} \sqrt{\frac{m E}{\hbar^{2}}}} & \text { for } D=3 \\ -\frac{2 \pi \hbar^{2}}{m} \frac{1}{\ln \left(a_{B} \sqrt{\frac{m E}{\hbar^{2}}} e^{\gamma} / 2\right)+i \pi / 2} & \text { for } D=2 \\ -\frac{2 \hbar^{2}}{m} \frac{i \sqrt{\frac{m E}{\hbar^{2}}}}{1+i a_{B} \sqrt{\frac{m E}{\hbar^{2}}}} & \text { for } D=1\end{cases}
$$

with $\gamma=0.577 \ldots$ the Euler-Mascheroni constant (Mora and Castin (2003); Girardeau, Nguyen and Olshanii (2004); Pricoupenko (2011)). 
In $3 \mathrm{D}$ one can safely set $E=0$, so that $T\left(0+i 0^{+}\right)=4 \pi \hbar^{2} a_{B} / m$, and Eq. (41) is immediately recovered. In $2 \mathrm{D}$ and $1 \mathrm{D}$ one must use Eq. (42) with a finite value of $E$, while in $1 \mathrm{D}$ one gets $T\left(E+i 0^{+}\right)=-2 \hbar^{2} /\left(m a_{B}\right)$ under the condition $a_{B} \sqrt{m E / \hbar^{2}} \gg 1$.

The second term in Eq. (41) is UV divergent, and this divergence may be regularized by introducing a hard cutoff $\Lambda$ for momenta (Momentum Cutoff Regularization, MCR). In this way one renormalizes $g=g(\Lambda)$ to reproduce the physical scattering length $a_{B}$ and lets $\Lambda$ go to infinity at the end of the calculation. This method will be discussed in detail in Subsection 3.3.

In Subsection 3.4 we shall see how one can recover Bogoliubov's result by introducing convergence factors into Eq. (32) and then using Eq. (41) to fully remove the divergence of quantum fluctuations (Convergence factor regularization, CFR).

Before discussing in details these methods, we illustrate a regularization procedure largely employed in the renormalization program of gauge theories: the method of Analytic or Dimensional Regularization (DR). We refer to Leibbrandt (1975) for a review.

\subsection{Dimensional regularization (DR)}

Analytic regularization exploits the concept of analytic continuation in some complex parameter. In its best known version, developed by 't Hooft and Veltman (1972), the parameter is the space (or space-time in the relativistic case) dimension $D$ and for this reason this procedure is also known as dimensional regularization.

In this section we illustrate the main ingredients of this method to get finite physical values from the otherwise divergent Eq. (15) for the ideal Bose gas and Eq. (39) for the interacting Bose gas.

We begin by recalling the fundamental 't Hooft and Veltman (1972) conjecture stating that in the context of dimensional regularization integrals over a polynomial identically vanish, i.e.

$$
\int_{0}^{+\infty} d q q^{D-1}\left(q^{2}\right)^{n-1}=0, \quad n=0,1,2 \ldots
$$

where $D$ may assume non integer values. The proof of this astonishing relation can be found in Sect IV of Leibbrandt (1975))). As a first consequence of Eq. (44) one gets from Eq. (41) in $D=3$

$$
g=\frac{4 \pi \hbar^{2} a_{B}}{m}
$$


implying that in dimensional regularization the strength $g$ of a 3D contact interaction is the bare scattering length $a_{B}$, apart from a multiplicative constant inversely proportional to the mass of the interacting particles.

As a second ingredient of dimensional regularization, one notices that for any complex $z$ with $\operatorname{Re}(z)>0$ one may write

$$
a^{-z}=\frac{1}{\Gamma(z)} \int_{0}^{+\infty} t^{z-1} e^{-a t}
$$

where

$$
\Gamma(z)=\int_{0}^{+\infty} t^{z-1} e^{-z}, \quad \operatorname{Re}(z)>0
$$

is Euler's Gamma function. To discuss situations where $\operatorname{Re}(z) \leq 0$, we must analytically continue the definition of Eq.(47) to the left part of the $z$ plane. Such continuation is found to be analytic everywhere except at the points where $z$ is a negative integer or zero. We will then treat such points with particular care.

Using Eq. (46) one gets immediately that Euler's beta function:

$$
B(x, y)=\int_{0}^{+\infty} d t \frac{t^{x-1}}{(1+t)^{x+y}}, \quad \operatorname{Re}(x), \operatorname{Re}(y)>0
$$

may be continued to complex values of $x$ and $y$ as (Ryder, 2001)

$$
B(x, y)=\frac{\Gamma(x) \Gamma(y)}{\Gamma(x+y)}
$$

Using Eq. (48) we may rewrite Eq. (39) for the zero point energy of a repulsive Bose gas in dimension $D$ as:

$$
\frac{\Omega_{g}^{(0)}}{L^{D}}=\frac{S_{D}(2 \mu)^{\frac{D}{2}+1}}{4(2 \pi)^{D}}\left(\frac{2 m}{\hbar^{2}}\right)^{\frac{D}{2}} B\left(\frac{D+1}{2},-\frac{D+2}{2}\right),
$$

Let us now analyze in detail the consequences of Eqs. (44) and (50).

\subsubsection{Ideal Bose gas - DR}

The zero point energy Eq. (15) of the Ideal Bose gas is an integral over a sum of poynomials in $q^{2}$. From 't Hooft and Veldman conjecture, Eq. (44), it follows that in dimensional regularization its value is zero in any of the spatial dimensions $D=1,2,3$

$$
\frac{\Omega^{(0)}}{L^{D}}=0
$$

As said before, the zero-point fluctuations of the ideal $D$-dimensional Bose gas are then fully removed by regularization and the exact grand potential is given by Eq. (16). 


\subsubsection{Repulsive Bose gas in $1 D$ and $3 D-D R$}

In the case of an interacting Bose gas, we rewrite Eq. (39) using Eq. (49) to get

$$
\frac{\Omega_{g}^{(0)}}{L^{D}}=\frac{S_{D}(2 \mu)^{\frac{D}{2}+1}}{4(2 \pi)^{D}}\left(\frac{2 m}{\hbar^{2}}\right)^{\frac{D}{2}} \frac{\Gamma\left(\frac{D+1}{2}\right) \Gamma\left(-\frac{D+2}{2}\right)}{\Gamma\left(-\frac{1}{2}\right)} .
$$

This expression is now finite when $D=1$ or $D=3$, while it is still divergent if $D=2$ since $\Gamma(p)$ diverges for integers $p \leq 0$.

For the repulsive Bose gas in $D=1$, from Eq. (52) we immediately find

$$
\frac{\Omega_{g}^{(0)}}{L}=-\frac{2}{3 \pi}\left(\frac{m}{\hbar^{2}}\right)^{\frac{1}{2}} \mu^{3 / 2} .
$$

The corresponding total grand potential thus reads

$$
\frac{\Omega}{L}=-\frac{\mu^{2}}{2 g}-\frac{2}{3 \pi}\left(\frac{m}{\hbar^{2}}\right)^{\frac{1}{2}} \mu^{3 / 2}+\frac{1}{\beta L} \sum_{\mathbf{q}} \ln \left(1-e^{-\beta E_{q}}\right) .
$$

One may show that in the range of validity of Bogoliubov approximation, i.e. $\gamma(D=1)=\frac{m}{\hbar^{2}} \frac{g}{n} \ll 1$ with $n$ the $1 \mathrm{D}$ number density, this expression agrees with the exact result obtained by Lieb and Liniger (1963). On the contrary, as a consequence of the failure of the Gaussian approximation, in the limit of large $\gamma$, i.e. small $n$, one does not recover the correct grand potential of the Tonks-Girardeau gas (Girardeau (1960)) at zero temperature, but obtains instead twice its value.

In the three-dimensional case, setting $D=3$ in Eq. (52) we get instead

$$
\frac{\Omega_{g}^{(0)}}{L^{3}}=\frac{8}{15 \pi^{2}}\left(\frac{m}{\hbar^{2}}\right)^{3 / 2} \mu^{5 / 2} \text {. }
$$

Notice that Gaussian quantum fluctuations, Eqs. (53) and (55), contribute with different signs to the grand-potentials in $1 \mathrm{D}$ and $3 \mathrm{D}$. The total grand potential of the three-dimensional Bose gas is then given by

$$
\frac{\Omega}{L^{3}}=-\frac{\mu^{2}}{2 g}+\frac{8}{15 \pi^{2}}\left(\frac{m}{\hbar^{2}}\right)^{3 / 2} \mu^{5 / 2}+\frac{1}{\beta L^{3}} \sum_{\mathbf{q}} \ln \left(1-e^{-\beta E_{q}}\right) .
$$

This is exactly the grand potential obtained by Lee. Huang and Yang (1957) by considering the contributions of the zero point energy of Bogoliubov (Bogoliubov, 1947) excitations and, contrary to the 1D case, it is reliable in the low-density regime (i.e. for $\gamma(D=3)=\frac{m}{\hbar^{2}} g n^{\frac{1}{3}} \ll 1$ ). 


\subsubsection{Repulsive Bose gas in 2D - DR}

Dimensional regularization of the repulsive Bose gas is more delicate in 2D. In fact, for $D=2 \mathrm{Eq}$. (52) diverges due to the presence of $\Gamma(-2)$. To cure this divergence, one then extends the calculation to non-integer dimension $D=2-\varepsilon$ and lets $\varepsilon$ go to zero at the end of the calculation. Eq. (52) can be written as

$$
\frac{\Omega_{g}^{(0)}}{L^{D}}=-\frac{m}{4 \pi \hbar^{2} \kappa^{\varepsilon}} \mu^{2} \Gamma\left(-2+\frac{\varepsilon}{2}\right),
$$

where the regulator $\kappa$ is an arbitrary scale wavenumber which enters for dimensional reasons. Since in the limit $\varepsilon \rightarrow 0$ one has:

$$
\Gamma\left(-2+\frac{\varepsilon}{2}\right)=\frac{1}{\varepsilon}+O\left(\varepsilon^{0}\right),
$$

to leading order in $1 / \varepsilon$ we get

$$
\frac{\Omega_{g}}{L^{D}}=-\frac{1}{2} \frac{m}{2 \pi \hbar^{2} \varepsilon \kappa^{\epsilon}} \mu^{2} .
$$

Comparing $\Omega_{g}^{(0)}$ with $\Omega_{0}$ in $D$ dimensions we conclude that

$$
\frac{\Omega_{0}}{L^{2}}+\frac{\Omega_{g}}{L^{2}}=-\frac{1}{2 g_{r}} \mu^{2},
$$

where the renormalized coupling constant $g_{r}$ given by

$$
\frac{1}{g_{r}}=\kappa^{\epsilon}\left(\frac{1}{g}+\frac{m}{2 \pi \hbar^{2} \kappa^{\varepsilon} \varepsilon}\right)
$$

The parameter $g_{r}$ is a "running coupling constant" for our theory which varies by changing $\kappa$. To extract its dependence on $\kappa$ within a renormalizationgroup scheme we obtain from (61) the differential flow equation

$$
\kappa \frac{d g_{r}}{d \kappa}=\frac{m}{2 \pi \hbar^{2}} g_{r}^{2}
$$

In the limit $\varepsilon \rightarrow 0$ we get the solution

$$
\frac{1}{g_{r}\left(\kappa_{0}\right)}-\frac{1}{g_{r}(\kappa)}=-\frac{m}{2 \pi \hbar^{2}} \ln \left(\frac{\kappa_{0}}{\kappa}\right) \text {. }
$$

By setting the Landau pole $(\mathrm{Kaku}, 1993)$ of Eq. (63) at the energy $\epsilon_{0}=$ $\hbar^{2} \kappa_{0}^{2} /(2 m)$, i.e. by defining

$$
\frac{1}{g_{r}\left(\kappa_{0}\right)}=0
$$


we obtain

$$
\frac{1}{g_{r}(\kappa)}=\frac{m}{4 \pi \hbar^{2}} \ln \left(\frac{\epsilon_{0}}{\mu}\right)
$$

when $\kappa$ is such that $\hbar^{2} \kappa^{2} /(2 m)=\mu$. Thus, the running coupling constant $g_{r}$ is indeed a function of the chemical potential $\mu$ and of an energy $\epsilon_{0}$.

By inserting the renormalized coupling constant $g_{r}(\kappa)$ from Eq. (65) into Eqs. (35) and (60) we thus obtain the regularized beyond-mean-field grand potential in the form

$$
\frac{\Omega}{L^{2}}=-\frac{m}{8 \pi \hbar^{2}} \ln \left(\frac{\epsilon_{0}}{\mu}\right) \mu^{2}+\frac{1}{\beta L^{2}} \sum_{\mathbf{q}} \ln \left(1-e^{-\beta E_{q}}\right) .
$$

This is exactly the equation of state derived by Popov (Popov, 1972) from a 2D hydrodynamic Hamiltonian taking into account the logarithmic behavior of the T-matrix that describes the scattering of two bosons (Schick, 1971). The energy $\epsilon_{0}$ appearing in Eq. (66) was introduced by Popov (Popov, 1972) as a cutoff in the T-matrix. By equating $g_{r}(\kappa)$ above with the $2 \mathrm{D}$ expression of $T(\mu)$ from Eq. (43) we identify $\epsilon_{0}$ as: $\epsilon_{0}=\hbar^{2} /\left(m a_{B}{ }^{2} e^{\gamma}\right)$.

Notice that any dependence on the bare interaction strength $g$ has completely disappeared from the final expression Eq. (66) of the grand potential.

\begin{tabular}{|c|c|}
\hline \hline Dimension & Grand potential \\
\hline$D=3$ & $\frac{\Omega}{L^{3}}=-\frac{\mu^{2}}{2 g}+\frac{8}{15 \pi^{2}}\left(\frac{m}{\hbar^{2}}\right)^{3 / 2} \mu^{5 / 2}+\frac{1}{\beta L^{3}} \sum_{\mathbf{q}} \ln \left(1-e^{-\beta E_{q}}\right)$ \\
\hline$D=2$ & $\frac{\Omega}{L^{2}}=-\frac{m}{8 \pi \hbar^{2}} \ln \left(\frac{\epsilon_{0}}{\mu}\right) \mu^{2}+\frac{1}{\beta L^{2}} \sum_{\mathbf{q}} \ln \left(1-e^{-\beta E_{q}}\right)$ \\
\hline$D=1$ & $\frac{\Omega}{L}=-\frac{\mu^{2}}{2 g}-\frac{2}{3 \pi}\left(\frac{m}{\hbar^{2}}\right)^{\frac{1}{2}} \mu^{3 / 2}+\frac{1}{\beta L} \sum_{\mathbf{q}} \ln \left(1-e^{-\beta E_{q}}\right)$ \\
\hline \hline
\end{tabular}

Table 1: Grand potential $\Omega$ of the $D$-dimensional interacting Bose gas, with Bogoliubov spectrum $E_{q}=\sqrt{\frac{\hbar^{2} k^{2}}{2 m}\left(\frac{\hbar^{2} k^{2}}{2 m}+2 \mu\right)}$, obtained after regularization of zero-point Gaussian fluctuations. $\mu$ is the chemical potential and $\beta=1 /\left(k_{B} T\right)$ with $k_{B}$ the Boltzmann constant and $T$ the absolute temperature.

For the sake of completeness, in Table 1 we report the final equation of state for $D=1,2,3$ of the interacting Bose gas at the gaussian level.

\subsection{Momentum-cutoff regularization (MCR)}

In this subsection we show how to regularize the divergent zero-point energy of the bosonic gas by means of an ultraviolet cutoff $\Lambda$ in the wavenumber 
$q$ and a subsequent renormalization of the bare parameters of the theory.

\subsubsection{Ideal Bose gas - MCR}

By using a high wavenumber cutoff $\Lambda$, the zero-temperature contribution of quantum fluctuations to the grand potential of the ideal Bose gas reported in Eq. (15) becomes

$$
\begin{gathered}
\frac{\Omega^{(0)}}{L^{D}}=\frac{1}{2} \frac{S_{D}}{(2 \pi)^{D}}\left(\frac{\hbar^{2}}{2 m} \int_{0}^{\Lambda} d q q^{D+1}-\mu \int_{0}^{\Lambda} d q q^{D-1}\right) \\
=\frac{1}{2} \frac{S_{D}}{(2 \pi)^{D}}\left(\frac{\hbar^{2}}{2 m(D+2)} \Lambda^{D+2}-\mu \int_{0}^{\Lambda} d q q^{D-1}\right) .
\end{gathered}
$$

The term proportional to $\Lambda^{D+2}$ is independent of $\mu$ and can therefore be ignored. The other term depends linearly on $\mu$ but it can be absorbed into $\frac{\Omega^{(0)}}{L^{D}}$ by redefining the order parameter $\psi_{0}$ in the zero-temperature grand potential

$$
\frac{\Omega_{0}}{L^{D}}+\frac{\Omega^{(0)}}{L^{D}}=-\mu \psi_{0}^{2}-\mu \frac{1}{2} \frac{S_{D}}{(2 \pi)^{D}} \int_{0}^{\Lambda} d q q^{D-1} .
$$

In fact, by setting

$$
\psi_{0}^{2}=\psi_{0, r}^{2}+\delta \psi_{0}^{2}
$$

with the counterterm

$$
\delta \psi_{0}^{2}=-\frac{1}{2} \frac{S_{D}}{(2 \pi)^{D}} \int_{0}^{\Lambda} d q q^{D-1}
$$

one finds the total grand potential in the form

$$
\frac{\Omega}{L^{D}}=-\mu \psi_{0, r}^{2}+\frac{1}{\beta L^{D}} \sum_{\mathbf{q}} \ln \left(1-e^{-\beta \xi_{q}}\right) .
$$

This is exactly Eq. (16), apart for the subscript $r$ in $\Omega$ and $\psi_{0}$. In Section II we have found the same grand potential by using dimensional regularization.

\subsubsection{Repulsive Bose gas in $1 D$ and 3D - MCR}

For the interacting Bose gas Eq. (39) gives

$$
\begin{aligned}
\frac{\Omega_{g}^{(0)}}{L^{D}} & =\frac{1}{2} \frac{S_{D}}{(2 \pi)^{D}} \int_{0}^{\Lambda} d q q^{D-1} \sqrt{\frac{\hbar^{2} q^{2}}{2 m}\left(\frac{\hbar^{2} q^{2}}{2 m}+2 \mu\right)} \\
& =\frac{S_{D}(2 \mu)^{\frac{D}{2}+1}}{4(2 \pi)^{D}}\left(\frac{2 m}{\hbar^{2}}\right)^{\frac{D}{2}} \int_{0}^{Z} d z z^{\frac{D-1}{2}} \sqrt{1+z}
\end{aligned}
$$


where $Z=\hbar^{2} \Lambda^{2} /(4 m \mu)$. These expressions are now well defined for $D=$ $1,2,3$ but obviously diverge as $Z \rightarrow+\infty$. As we shall see however, also in this case the divergent contributions may be removed by introducing appropriate counterterms (Feynman, 1948; Kaku, 1993; Schakel, 2008) which renormalize the bare parameters of the theory.

For the one-dimensional repulsive Bose gas we set $D=1$ in Eq. (72) and after integration we find

$$
\frac{\Omega_{g}^{(0)}}{L}=\frac{2}{3 \pi}\left(\frac{m}{\hbar^{2}}\right)^{1 / 2} \mu^{3 / 2}\left(-1+(1+Z)^{3 / 2}\right) .
$$

In the large- $\Lambda$ limit, the zero-temperature total grand potential is then given by

$$
\begin{aligned}
\frac{\Omega_{0}}{L}+\frac{\Omega_{g}^{(0)}}{L} & =-\frac{\mu^{2}}{2 g}-\frac{2}{3 \pi}\left(\frac{m}{\hbar^{2}}\right)^{1 / 2} \mu^{3 / 2}+\frac{\hbar^{2}}{12 \pi m} \Lambda^{3} \\
& +\frac{\mu}{2 \pi} \Lambda+O\left(\frac{1}{\Lambda}\right) .
\end{aligned}
$$

The term proportional to $\Lambda^{3}$ is independent of $\mu$ and can then be ignored. The term proportional to $\Lambda$ which depends on $\mu$ may be absorbed by redefining the bare chemical potential appearing in the original zero-temperature mean-field grand-potential. By defining

$$
\mu_{r}=\mu-\delta \mu
$$

with

$$
\delta \mu=\frac{g \Lambda}{2 \pi}
$$

Eq (74) becomes:

$$
\frac{\Omega}{L}=-\frac{\mu_{r}^{2}}{2 g}-\frac{2}{3 \pi}\left(\frac{m}{\hbar^{2}}\right)^{1 / 2} \mu_{r}^{3 / 2}+\frac{1}{\beta L} \sum_{\mathbf{q}} \ln \left(1-e^{-\beta E_{q}}\right),
$$

which indeed coincides Eq. (54) obtained with dimensional regularization if one identifies $\mu_{r}$ with the measured chemical potential.

For the three-dimensional interacting Bose gas we set $D=3$ in Eq. (72) and after integration we find

$$
\frac{\Omega_{g}^{(0)}}{L^{3}}=\frac{8}{15 \pi^{2}}\left(\frac{m}{\hbar^{2}}\right)^{3 / 2} \mu^{3 / 2}\left(1+\frac{1}{2} \sqrt{1+Z}\left(3 Z^{2}+Z-2\right)^{3 / 2}\right),
$$


where $Z=\hbar^{2} \Lambda^{2} /(4 m \mu)$. The zero-temperature total grand potential is then given by

$$
\begin{aligned}
\frac{\Omega_{0}}{L^{3}}+\frac{\Omega_{g}^{(0)}}{L} & =-\frac{\mu^{2}}{2 g}+\frac{8}{15 \pi^{2}}\left(\frac{m}{\hbar^{2}}\right)^{3 / 2} \mu^{5 / 2}-\frac{7 \hbar^{2}}{1280 \sqrt{2} \pi m} \Lambda^{5} \\
& +\frac{\mu}{12 \pi^{2}} \Lambda^{3}-\frac{m \mu^{2}}{4 \pi^{2} \hbar^{2}} \Lambda+O\left(\frac{1}{\Lambda}\right),
\end{aligned}
$$

in the large- $\Lambda$ limit. The term proportional to $\Lambda^{5}$ is independent of $\mu$ and can be ignored. Since now there are two relevant (containing $\mu$ ) divergent terms, we need to renormalize both parameters $\mu$ and $g$ to absorb them into the mean-field form. As reported by Schakel (Schakel, 2008), this renormalization of the bare physical parameters is achieved by setting

$$
\begin{aligned}
& \mu_{r}=\mu-\frac{g \Lambda^{3}}{12 \pi^{2}} . \\
& g_{r}=g-\frac{m g^{2} \Lambda}{2 \pi^{2} \hbar^{2}}
\end{aligned}
$$

so that the renormalized grand potential becomes

$$
\frac{\Omega}{L^{3}}=-\frac{\mu_{r}^{2}}{2 g_{r}}-\frac{8}{15 \pi^{2}}\left(\frac{m}{\hbar^{2}}\right)^{3 / 2} \mu_{r}^{5 / 2}+\frac{1}{\beta L^{3}} \sum_{\mathbf{q}} \ln \left(1-e^{-\beta E_{q}}\right),
$$

which is equivalent to the equation of state Eq. (56) previously obtained through dimensional regularization.

It is worth noticing that the result (81) obtained by redefining the parameters of the theory $\mu$ and $g$ so that Eq. (79) takes the mean-field form, coincides with the expression obtained from Eq. (42) with a high wavenumber cutoff $\Lambda$.

\subsubsection{Repulsive Bose gas in 2D - MCR}

The momentum-cutoff regularization of the two-dimensional repulsive Bose gas requires a very careful analysis. We first set $D=2$ into Eq. (72) and after integration we find

$$
\frac{\Omega_{g}^{(0)}}{L^{2}}=\frac{m}{4 \pi \hbar^{2}} \mu^{2}\left(\frac{2 Z^{3}+3 Z^{2}+Z}{\sqrt{Z^{2}+Z}}-\ln (2 \sqrt{Z})\right),
$$


where $Z=\hbar^{2} \Lambda^{2} /(4 m \mu)$. The zero-temperature total grand potential is then given by

$$
\begin{aligned}
\frac{\Omega_{0}}{L^{2}}+\frac{\Omega_{g}^{(0)}}{L^{2}} & =-\frac{\mu^{2}}{2 g}+\frac{m}{16 \pi \hbar^{2}} \mu^{2}+\frac{\hbar^{2}}{32 \pi m} \Lambda^{4}+\frac{\mu}{8 \pi} \Lambda^{2} \\
& -\frac{m}{8 \pi \hbar^{2}} \mu^{2} \ln \left(\frac{\hbar^{2} \Lambda^{2}}{m \mu}\right)+O\left(\frac{1}{\Lambda^{4}}\right),
\end{aligned}
$$

in the large- $\Lambda$ limit. The term proportional to $\Lambda^{4}$ is independent of $\mu$ and can be ignored. The terms proportional to $\Lambda^{2}$ and $\ln \left(\Lambda^{2}\right)$ depend on $\mu$ and therefore they must be properly treated. First of all, we separate terms proportional to integer powers of $\mu$ from a term containing a logarithmic dependence by writing

$$
\ln \left(\frac{\hbar^{2} \Lambda^{2}}{m \mu}\right)=\ln \left(\frac{\hbar^{2} \Lambda^{2}}{m \epsilon_{c}}\right)+\ln \left(\frac{\epsilon_{c}}{\mu}\right)
$$

where the energy $\epsilon_{c}$ is completely arbitrary. Now we can absorb the divergent terms by setting

$$
\begin{aligned}
& \mu_{r}=\mu-\frac{\frac{g \Lambda^{2}}{8 \pi}}{1-\frac{m g}{4 \pi \hbar^{2}} \ln \frac{\varepsilon_{c}}{\mu}} . \\
& g_{r}=g-\frac{m g^{2}}{4 \pi \hbar^{2}} \ln \left(\frac{\hbar^{2} \Lambda^{2}}{m \epsilon_{c}}\right) .
\end{aligned}
$$

to obtain the renormalized total grand potential

$$
\frac{\Omega}{L^{2}}=-\frac{\mu_{r}^{2}}{2 g_{r}}-\frac{m}{8 \pi \hbar^{2}} \mu_{r}^{2} \ln \left(\frac{\epsilon_{c}}{e \mu_{r}}\right)+\frac{1}{\beta L^{2}} \sum_{\mathbf{q}} \ln \left(1-e^{-\beta E_{q}}\right),
$$

which can finally be rewritten as

$$
\frac{\Omega}{L^{2}}=-\frac{m}{8 \pi \hbar^{2}} \mu_{r}^{2} \ln \left(\frac{\epsilon_{0}}{\mu_{r}}\right)+\frac{1}{\beta L^{2}} \sum_{\mathbf{q}} \ln \left(1-e^{-\beta E_{q}}\right),
$$

where

$$
\epsilon_{0}=\epsilon_{c} e^{4 \pi \hbar^{2} /\left(g_{r} m\right)-1 / 2} .
$$

Again the renormalized coupling constant coincides with the expression obtained from the scattering theory, Eq. (42), with a high wavenumber cutoff $\Lambda$.

Also in this two-dimensional case the total grand potential obtained with the cutoff regularization, Eq. (89), is the same as the one found with dimensional-regularization, Eq. (66). 


\subsection{Convergence-factor regularization (CFR)}

We now analyze a third method of regularization of zero-point fluctuations. This method, mainly used in condensed-matter physics (Nagaosa, 1999; Stoof et al., 2009; Altland and Simons, 2010), is based on the use of a convergence factor $e^{i \omega_{n} 0^{+}}$when performing the Matsubara sums in Eqs. (8) and (32). As we shall see, in the case of the ideal Bose gas the presence of the convergence factor removes completely the zero-point energy of quantum fluctuations, while in the case of the interacting Bose gas the cancellation of the zero-point divergence is only partial.

\subsubsection{Ideal Bose gas - CFR}

As explained in detail by Altland and Simons (2010), the inclusion of a convergence factor $e^{i \omega_{n} 0^{+}}$into Eq. (8) exactly produces, after the complex integration associated to the Matsubara sum, a counterterm which removes the zero-point divergence, namely Eq. (10) is modified into

$$
\frac{1}{2 \beta} \sum_{n=-\infty}^{+\infty} \ln \left[\beta^{2}\left(\hbar^{2} \omega_{n}^{2}+\xi_{q}^{2}\right)\right] e^{i \omega_{n} 0^{+}}=\frac{1}{\beta} \ln \left(1-e^{-\beta \xi_{q}}\right),
$$

where $e^{i \omega_{n} 0^{+}}$means $\lim _{\delta \rightarrow 0^{+}} e^{i \omega_{n} \delta}$. Consequently, the grand potential is given by Eq. (16), as it must.

\subsubsection{Repulsive Bose gas in $1 D$ and $3 D-C F R$}

In the case of the repulsive Bose gas the introduction of a convergencefactor into Eq. (32) leads to (Diener et al., 2008)

$$
\begin{array}{r}
\frac{1}{2 \beta} \sum_{n=-\infty}^{+\infty} \ln \left[\beta^{2}\left(\hbar^{2} \omega_{n}^{2}+E_{q}^{2}\right)\right] e^{i \omega_{n} 0^{+}} \\
=\frac{1}{2}\left(\frac{E_{q}}{2}-\frac{\hbar^{2} q^{2}}{2 m}-\mu\right)+\frac{1}{\beta} \ln \left(1-e^{-\beta \xi_{q}}\right) .
\end{array}
$$

As a consequence, in the continuum limit the zero-temperature grand potential reads

$$
\frac{\Omega_{0}}{L^{D}}+\frac{\Omega_{g}^{(0)}}{L^{D}}=-\frac{\mu^{2}}{2 g}+\frac{1}{2} \int \frac{d^{D} \mathbf{q}}{(2 \pi)^{D}}\left(E_{q}-\frac{\hbar^{2} q^{2}}{2 m}-\mu\right),
$$

where $E_{q}$ is given by Eq. (38).

In $1 \mathrm{D}$, after integration one finds

$$
\frac{\Omega_{g}^{(0)}}{L}=-\frac{2}{3 \pi}\left(\frac{m}{\hbar^{2}}\right)^{1 / 2} \mu^{3 / 2}
$$


confirming the equation of state (54) obtained above by both the dimensional and momentum-cutoff regularization.

In the three-dimensional case the integral of Eq. (93) is ultraviolet divergent. As we have previously seen, this kind of divergence can be fully removed by dimensional regularization or, equivalently, by momentum-cutoff regularization. Moreover, it can also be removed by taking into account scattering theory at the second order, namely Eq. (41), which in the continuum limit and $D=3$ may be written as

$$
\frac{1}{g_{r}}=\frac{1}{g}+\int \frac{d^{3} \mathbf{q}}{(2 \pi)^{3}} \frac{m}{\hbar^{2} q^{2}}
$$

with $g_{r}=4 \pi \hbar^{2} a_{B} / m$. The integral in the right hand side of Eq. (95) is ultraviolet divergent, but by inserting Eq. (95) into Eq. (93) one obtains

$$
\begin{aligned}
\frac{\Omega_{0}}{L^{3}} & +\frac{\Omega_{g}^{(0)}}{L^{3}}=-\frac{\mu^{2}}{2 g_{r}} \\
& +\frac{1}{2} \int \frac{d^{3} \mathbf{q}}{(2 \pi)^{D}}\left(E_{q}-\frac{\hbar^{2} q^{2}}{2 m}-\mu+\frac{m \mu^{2}}{\hbar^{2} q^{2}}\right) .
\end{aligned}
$$

This integral is now finite and the zero-temperature grand potential once again is found to be

$$
\frac{\Omega_{0}}{L^{3}}+\frac{\Omega_{g}^{(0)}}{L^{3}}=-\frac{\mu^{2}}{2 g_{r}}+\frac{8}{15 \pi^{2}}\left(\frac{m}{\hbar^{2}}\right)^{3 / 2} \mu^{5 / 2} .
$$

\subsubsection{Repulsive Bose gas in 2D - CFR}

Also in the two-dimensional case the integral of Eq. (93) is ultraviolet divergent. Proceeding as in the 3D case with scattering theory at the second order, i.e. by using the 2D version of Eq. (95) given by

$$
\frac{1}{g_{r}}=\frac{1}{g}+\int \frac{d^{2} \mathbf{q}}{(2 \pi)^{2}} \frac{m}{\hbar^{2} q^{2}},
$$

leads to the zero-temperature grand potential

$$
\begin{aligned}
\frac{\Omega_{0}}{L^{2}} & +\frac{\Omega_{g}^{(0)}}{L^{2}}=-\frac{\mu^{2}}{2 g_{r}} \\
& +\frac{1}{2} \int \frac{d^{2} \mathbf{q}}{(2 \pi)^{D}}\left(E_{q}-\frac{\hbar^{2} q^{2}}{2 m}-\mu+\frac{m \mu^{2}}{\hbar^{2} q^{2}}\right)
\end{aligned}
$$


Here the ultraviolet divergence has been cancelled but the last term introduces an infrared divergence which must be cured by introducing a lowenergy cutoff $\epsilon_{c}$ (and its corresponding wavenumber $k_{c}=\left(m \epsilon_{c}\right)^{1 / 2} / \hbar$ ). In this way, we find

$$
\frac{\Omega_{g}^{(0)}}{L^{2}}=\frac{m}{4 \pi \hbar^{2}} \mu^{2}\left(\frac{1}{4}-\ln 2-\frac{1}{2} \ln \frac{\epsilon_{c}}{4 \mu}\right),
$$

which, after some algebraic manipulations, gives back the total grand potential of Eq. (89), with Eq. (90) and $\mu$ instead of $\mu_{r}$.

In conclusion, also for the two-dimensional interacting Bose gas the three methods of regularization (dimensional, momentum-cutoff, and convergencefactor) of divergent Gaussian fluctuations give rise to the same equation of state. However, in 2D the convergence-factor regularization plus scattering theory needs also a further momentum-cutoff regularization of the residual infrared divergence.

\section{Experiments vs theory for bosonic superfluids}

\subsection{Repulsive Bose gas in $3 D$}

Despite the very large number of experiments with bosonic gases made of dilute and ultracold alkali-metal atoms, only in recent years zero-point energy (i.e. beyond-mean-field) effects have been measured.

In 2008 Papp et al. (2008) got informations on the zero-temperature chemical potential $\mu$ of a gas of ${ }^{85} \mathrm{Rb}$ atoms as a function of the gas parameter $n a_{B}^{3}$. Through the Feschbach resonance technique, they tuned the scattering length $a_{B}$ of their harmonically trapped atomic sample up to $1000 a_{0}\left(a_{0}=0.53 \cdot 10^{-8} \mathrm{~cm}\right.$ is the Bohr radius). Since their sample had a mean number density $n=7.6 \cdot 10^{-13} \mathrm{~cm}^{-3}$ they were then varying the gas parameter $n a_{B}{ }^{3}$ up to 0.002. Using two-photon Bragg spectroscopy to probe the Bogoliubov excitation spectrum, see Eq. (38), of the strongly interacting 3D Bose-Einstein condensate, they then measured the energy required to promote an atom out of the condensate less the bare kinetic energy, namely:

$$
E_{q}-\frac{\hbar^{2} q^{2}}{2 m}=\sqrt{\frac{\hbar^{2} q^{2}}{2 m}\left(\frac{\hbar^{2} q^{2}}{2 m}+2 \mu\right)}-\frac{\hbar^{2} q^{2}}{2 m} .
$$

In the regime of high-momentum excitations this quantity becomes

$$
E_{q}-\frac{\hbar^{2} q^{2}}{2 m} \simeq \mu
$$


Thus, measuring $E_{q}-\hbar^{2} q^{2} /(2 m)$ at high momenta Papp et al. (2008) determined the behavior of $\mu$ hence confirming the contributions of quantum fluctuations as discussed above.

In fact, by using the one-loop grand potential given by (56) and the thermodynamic formula (19) one immediately finds at zero temperature

$$
n=\frac{\mu}{g}-\frac{4}{3 \pi^{2}}\left(\frac{m}{\hbar^{2}}\right)^{3 / 2} \mu^{3 / 2} .
$$

¿From this equation one can easily determine $\mu$ as a function of $n$ and $a_{B}$, that is

$$
\mu=\mu_{0}+\mu_{g}
$$

where

$$
\mu_{0}=\frac{4 \pi \hbar^{2}}{m} a_{B} n
$$

is the mean-field result with $g=4 \pi \hbar^{2} a_{B} / m$ and

$$
\mu_{g}=\frac{4 \pi \hbar^{2}}{m} a_{B} n\left(\frac{32}{\sqrt{\pi}}\left(n a_{B}^{3}\right)^{1 / 2}\right)
$$

is the zero-temperature Gaussian correction, under the condition $n a_{B}^{3} \ll 1$.

In 2012 Wild et al. (2012) found beyond-mean-field effects in the zerotemperature equation of state of a repulsive 3D Bose gas made of $N \simeq 6 \cdot 10^{4}$ ${ }^{85} \mathrm{Rb}$ atoms by measuring the two-body contact $C_{2}$. $C_{2}$ is an extensive thermodynamic quantity that is connected to the derivative of the total energy $E$ of the system with respect to the s-wave scattering length $a_{B}$ (Tan (2008)), namely

$$
C_{2}=\frac{8 \pi m a_{B}^{2}}{\hbar^{2}} \frac{d E}{d a_{B}} .
$$

The contact $C_{2}$ characterizes the tail of the momentum distribution of a many-body system of identical particles (Tan (2008)) and also the tail of the rate $\Gamma(\omega)$ for inducing transitions between spin states in rf spectroscopy (Wild et al. (2012)). Indeed, in the experiment a pulse of radio-frequency $\omega$ was used to drive a Zeeman transition and to transfer a small fraction of spinpolarized bosonic atoms into another spin state. Then, from the observed $\Gamma(\omega)$, Wild et al. (2012) extracted the value of the two-body contact $C_{2}$. In Fig. 3 we plot their experimental data for $\frac{C_{2}}{N}$ vs $a_{B}$ as filled circles. In the figure the dashed line is the mean-field value of $\frac{C_{2}}{N}$, that is

$$
\frac{C_{2}}{N}=16 \pi^{2} n a_{B}^{2}
$$




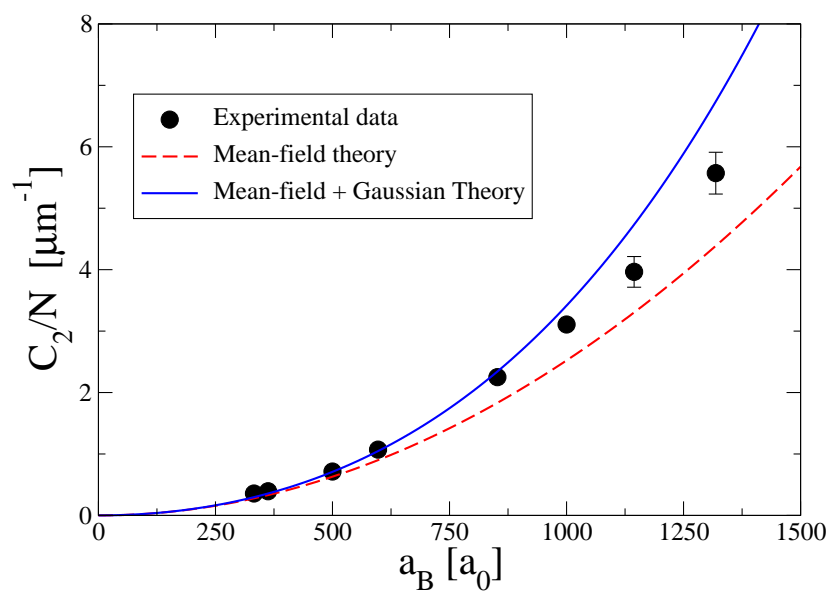

Figure 3: Contact $C_{2}$ vs scattering length $a_{B}$ for the zero-temperature repulsive Bose gas. Comparison between mean-field theory (dashed line) and one-loop theory, i.e mean-field plus Gaussian fluctuations (solid line). Filled circles are experimental data of Wild et al. (2012), obtained with a ${ }^{85} \mathrm{Rb}$ atomic condensate using rf spectroscopy, where $n=5.8 \cdot 10^{12}$ $\mathrm{cm}^{-3}$ is the mean number density and the gas parameter $n a_{B}^{3}$ reaches a maximum of 0.0018. The scattering length $a_{B}$ is in units of the Bohr radius, $a_{0}=0.53 \cdot 10^{-8} \mathrm{~cm}$.

while the solid line is the one-loop value of $\frac{C_{2}}{N}$, given by

$$
\frac{C_{2}}{N}=16 \pi^{2} n a_{B}^{2}\left(1+\frac{5}{2} \frac{128}{15 \sqrt{\pi}} \sqrt{n a_{B}^{3}}\right) .
$$

Both Eq. (108) and Eq. (109) are obtained from Eq. (107) and $E=\Omega+\mu N$ with the pertinent $\Omega$. As noticed in Wild et al. (2012), Fig. 3, confirms that by increasing the scattering length $a_{B}$ the two-body contact $C_{2}$ deviates from the mean-field prediction and the one-loop theory (mean-field plus Gaussian fluctuations) better reproduces the experimental data.

\subsection{Repulsive Bose gas in 2D}

In 2011 the thermodynamics of a 2D Bose gas was investigated by Yefsah et al. (2011) with ${ }^{87} \mathrm{Rb}$ atoms. As in previous thermodynamic investigations of a 3D Bose gas of ${ }^{7} \mathrm{Li}$ atoms (Nascimbene et al. $(2010)$ ), the analysis of Yefsah et al. (2011) was performed by analyzing in situ measurements of the density profiles $n(\mathbf{r})$ to extract the pressure $P(\mu, T)$ of the equivalent homogeneous gas.

The idea is the following: starting from Eq. (66), which is the 2D grand 
potential $\Omega$ of the uniform system, the pressure $P=-\Omega / L^{2}$ reads

$$
P(\mu, T)=\frac{m}{8 \pi \hbar^{2}} \ln \left(\frac{\epsilon_{0}}{\mu}\right)-k_{B} T \int \frac{d^{2} \mathbf{q}}{(2 \pi)^{2}} \ln \left(1-e^{-\sqrt{\frac{\hbar^{2} q^{2}}{2 m}\left(\frac{\hbar^{2} q^{2}}{2 m}+2 \mu\right)} /\left(k_{B} T\right)}\right)
$$

while the corresponding number density $n$ is given by

$$
n(\mu, T)=\left(\frac{\partial P}{\partial \mu}\right)_{T} .
$$

Using the local density approximation

$$
\mu \rightarrow \bar{\mu}-U(\mathbf{r})
$$

where $U(\mathbf{r})$ is the space-dependent confining potential and $\bar{\mu}$ is the chemical potential of the non homogeneous system, one gets the local number density $n(\mathbf{r})$ as

$$
n(\mathbf{r})=n(\mu=\bar{\mu}-U(\mathbf{r}), T) .
$$

Reversing this procedure, from the local number density $n(\mathbf{r})$ of the non homogeneous system one gets the pressure of the homogeneous system. The experimental data of $P(\mu, T)$ extracted by Yefsah et al. (2011) for the trapped gas of ${ }^{87} \mathrm{Rb}$ atoms suggest that the finite-temperature contribution to the pressure given by Eq. (110) is fully reliable. However, due to difficulties to reach extremely low temperatures, in this experiment they were not able to test the zero-temperature logarithmic behavior of the $2 \mathrm{D}$ equation of state. This important experimental investigation has been recently performed by Makhalov et al. (2014) on a 2D system of composite bosons made of bound pairs of ${ }^{6} \mathrm{Li}$ atoms. We shall discuss in detail this experiment in Section 6.2, comparing it with our theoretical predictions for attractive fermions in the deep BEC regime of the 2D BCS-BEC crossover.

\subsection{Repulsive Bose gas in 1D}

In 2004 Kinoshita et al. (Kinoshita et al. (2004)) reported the observation of a one-dimensional gas of cold rubidium- 87 atoms confined in a cigar shaped regions by two orthogonal strong light traps and moving almost freely in the third direction. In the experiment the 1D interaction strength $g_{1 D}$ is modified by changing the width $a_{\perp}$ of the transverse harmonic confinement, as $g_{1 D}=g_{3 D} /\left(2 \pi a_{\perp}^{2}\right)$ where, as said above, $g_{3 D}=4 \pi \hbar^{2} a_{3 D} / \mathrm{m}$. By changing the trap intensities and hence the atomic interaction strength $\gamma=\left(m / \hbar^{2}\right)\left(g_{1 D} / n_{1 D}\right)$ the atoms were made to act either like a Bose-Einstein condensate, when $\gamma \ll 1$ or like a Tonks-Girardeau gas of impenetrable 
bosons when $\gamma \gg 1$. Kinoshita et al. (Kinoshita et al. (2004)) thus measured the total 1D energy and the length of the gas. With no free parameters and over a wide range of coupling strengths, their data fit the exact solution for the ground state of a 1D Bose gas as found by Lieb and Liniger (1963).

This experiment confirms that in the weak-coupling regime $(\gamma \ll 1)$ the $1 \mathrm{D}$ system is a quasi-condensate very well described by the one-loop grand potential (54).

In the strong coupling regime, also studied with a completely different experimental setup by Paredes et al. (2004), the 1D system is a TonksGirardeau gas, whose grand potential is given by

$$
\frac{\Omega}{L}=-\frac{2 \sqrt{2}}{3 \pi}\left(\frac{m}{\hbar^{2}}\right)^{1 / 2} \mu^{3 / 2}
$$

and the corresponding chemical potential reads

$$
\mu=\frac{\pi^{2} \hbar^{2}}{2 m} n^{2}
$$

The failure in this case of the one-loop Gaussian approximation, which at $T=0$ gives the grand potential $\Omega$ of Eq.(54) instead of Eq.(114), is not surprising since Gaussian results are expected to be fully reliable only in the week-coupling regime.

\section{Functional integration for fermionic superfluids}

We have seen that after regularization, the zero-point energy of the Gaussian quantum fluctuations contributes a non-trivial term to the equation of state of an interacting $D$-dimensional Bose superfluid. Three different regularization approaches (dimensional regularization, momentum-cutoff regularization and convergence-factor regularization) produce the same finite result, which is however dependent on the dimensionality of the system. Moreover, at variance with both dimensional regularization and momentumcutoff regularization which are self-contained, convergence-factor regularization explicitly needs scattering theory (or, again, dimensional or cutoff regularization).

Extremely interesting is the study of the divergent zero-point energy of a $D$-dimensional two-spin-component Fermi superfluid in the BCS-BEC crossover. As will be discussed in detail below, the crossover from the weakly-paired Bardeen-Cooper-Schrieffer (BCS) state to the Bose-Einstein condensate (BEC) of molecular dimers has been experimentally achieved using ultracold fermionic alkali-metal atoms a few years ago in 3D (Greiner et al., 
2003; Chin et al., 2004) and quite recently recently also in a two-dimensional configuration (Makhalov et al., 2014). In the gas of paired fermions there are two kinds of elementary excitations: fermionic single-particle excitations with energy

$$
E_{s p}(k)=\sqrt{\left(\frac{\hbar^{2} k^{2}}{2 m}-\mu\right)^{2}+\Delta_{0}^{2}},
$$

where $\Delta_{0}$ is the pairing gap, and bosonic collective excitations with energy

$$
E_{c o l}(q)=\sqrt{\frac{\hbar^{2} q^{2}}{2 m}\left(\lambda \frac{\hbar^{2} q^{2}}{2 m}+2 m c_{B}^{2}\right)},
$$

where $\lambda \neq 0$ gives the first correction to the familiar low-momentum dispersion $E_{c o l}(q) \simeq c_{B} \hbar q$. Eq. (117) is obtained in the limit of a smallwavenumber $q$ from Gaussian fluctuations (Randeria et al., 1990; Marini et al., 1998). Notice that for a given scattering length both $\lambda$ and $c_{B}$ depend on the chemical potential $\mu$ and so does the energy gap $\Delta_{0}$. As we shall see, after regularization, the zero-point energy of these elementary excitations gives a relevant contribution to the equation of state of the fermionic superfluid.

Starting from the familiar BCS Lagrangian density of paired (attractive) fermions (Nagaosa, 1999)

$$
\mathscr{L}=\bar{\psi}_{s}\left[\hbar \partial_{\tau}-\frac{\hbar^{2}}{2 m} \nabla^{2}-\mu\right] \psi_{s}+g \bar{\psi}_{\uparrow} \bar{\psi}_{\downarrow} \psi_{\downarrow} \psi_{\uparrow}
$$

where $\psi_{s}(\mathbf{r}, \tau)$ and $\bar{\psi}_{s}(\mathbf{r}, \tau)$ are Grassman variables describing the fermionic field and $g<0$ is the strength of the s-wave inter-atomic coupling, the partition function $\mathcal{Z}$ of the uniform fermionic system in a $D$-dimensional volume $L^{D}$, and with chemical potential $\mu$ reads

$$
\mathcal{Z}=\int \mathcal{D}\left[\psi_{s}, \bar{\psi}_{s}\right] \exp \left\{-\frac{1}{\hbar} S\left[\psi_{s}, \bar{\psi}_{s}\right]\right\}
$$

where

$$
S\left[\psi_{s}, \bar{\psi}_{s}\right]=\int_{0}^{\hbar \beta} d \tau \int_{L^{D}} d^{D} \mathbf{r} \mathscr{L}\left(\psi_{s}, \bar{\psi}_{s}\right)
$$

is the Euclidean action functional. Through the exact Hubbard-Stratonovich transformation (Nagaosa (1999)) the Lagrangian density $\mathcal{L}$, quartic in the fermionic fields, can be rewritten as a quadratic form by introducing the auxiliary complex scalar field $\Delta(\mathbf{r}, \tau)$, namely

$$
\mathscr{L}_{e}=\bar{\psi}_{s}\left[\hbar \partial_{\tau}-\frac{\hbar^{2}}{2 m} \nabla^{2}-\mu\right] \psi_{s}+\bar{\Delta} \psi_{\downarrow} \psi_{\uparrow}+\Delta \bar{\psi}_{\uparrow} \bar{\psi}_{\downarrow}-\frac{|\Delta|^{2}}{g} .
$$


In this way the partition function $\mathcal{Z}$ of the fermionic system can be rewritten exactly as

$$
\mathcal{Z}=\int \mathcal{D}\left[\psi_{s}, \bar{\psi}_{s}\right] \mathcal{D}[\Delta, \bar{\Delta}] \exp \left\{-\frac{S_{e}\left[\psi_{s}, \bar{\psi}_{s}, \Delta, \bar{\Delta}\right]}{\hbar}\right\}
$$

where

$$
S_{e}\left[\psi_{s}, \bar{\psi}_{s}, \Delta, \bar{\Delta}\right]=\int_{0}^{\hbar \beta} d \tau \int_{L^{D}} d^{D} \mathbf{r} \mathscr{L}_{e}\left(\psi_{s}, \bar{\psi}_{s}, \Delta, \bar{\Delta}\right)
$$

is the the (exact) effective Euclidean action. Notice that now there is a functional integration also over $\Delta(\mathbf{r}, \tau)$. This is the price to pay for having an effective Lagrangian that is quadratic, instead of quartic, in the fermionic fields $\psi_{s}(\mathbf{r}, \tau)$ and $\bar{\psi}_{s}(\mathbf{r}, \tau)$.

The effect of fluctuations of the field $\Delta(\mathbf{r}, t)$ around its mean-field value $\Delta_{0}$ (the pairing gap) may be analyzed at the Gaussian level by taking

$$
\Delta(\mathbf{r}, \tau)=\Delta_{0}+\eta(\mathbf{r}, \tau),
$$

where $\eta(\mathbf{r}, \tau)$ is the complex pairing field of bosonic fluctuations. In particular, we are interested in the one-loop grand potential $\Omega$, given by

$$
\Omega=-\frac{1}{\beta} \ln (\mathcal{Z}) \simeq-\frac{1}{\beta} \ln \left(\mathcal{Z}_{m f} \mathcal{Z}_{g}\right)=\Omega_{m f}+\Omega_{g},
$$

where

$$
\mathcal{Z}_{m f}=\int \mathcal{D}\left[\psi_{s}, \bar{\psi}_{s}\right] \exp \left\{-\frac{S_{e}\left[\psi_{s}, \bar{\psi}_{s}, \Delta_{0}\right]}{\hbar}\right\}
$$

is the mean-field partition function and

$$
\mathcal{Z}_{g}=\int \mathcal{D}\left[\psi_{s}, \bar{\psi}_{s}\right] \mathcal{D}[\eta, \bar{\eta}] \exp \left\{-\frac{S_{g}\left[\psi_{s}, \bar{\psi}_{s}, \eta, \bar{\eta}, \Delta_{0}\right]}{\hbar}\right\}
$$

is the partition function of Gaussian pairing fluctuations, i.e. neglecting cubic and quartic contributions of $\eta$. Thus, one may write the total oneloop grand potential as

$$
\Omega=\Omega_{m f}+\Omega_{g}
$$

In Eq. (128)

$$
\Omega_{m f}=\Omega_{0}+\Omega_{m f}^{(0)}+\Omega_{m f}^{(T)}
$$

is the so-called mean-field grand potential, which includes the grand potential of the order parameter $\Delta_{0}$

$$
\Omega_{0}=-\frac{\Delta_{0}^{2}}{g} L^{D}
$$


the zero-point energy of fermionic single-particle excitations

$$
\Omega_{m f}^{(0)}=-\sum_{\mathbf{k}} E_{s p}(k)
$$

and the finite-temperature grand potential of the fermionic single-particle excitations

$$
\Omega_{m f}^{(T)}=\frac{2}{\beta} \sum_{\mathbf{k}} \ln \left(1+e^{-\beta E_{s p}(k)}\right) .
$$

In addition,

$$
\Omega_{g}=\Omega_{g}^{(0)}+\Omega_{g}^{(T)},
$$

is the grand potential of the bosonic Gaussian fluctuations, which includes the zero-point energy of bosonic collective excitations

$$
\Omega_{g}^{(0)}=\frac{1}{2} \sum_{\mathbf{q}} E_{c o l}(q),
$$

and their finite-temperature contribution

$$
\Omega_{g}^{(T)}=\frac{1}{\beta} \sum_{\mathbf{q}} \ln \left(1-e^{-\beta E_{c o l}(q)}\right) .
$$

Clearly both $\Omega_{m f}^{(0)}$ and $\Omega_{g}^{(0)}$ are ultraviolet divergent in the dimensions $D=1,2,3$. Regularization of these divergent terms is now complicated by the presence of the BCS-BEC crossover. Very recently we have obtained interesting analytical results in the BEC regime of the BCS-BEC crossover by removing the divergences of single-particle and collective excitations both in 3D (Salasnich and Bighin, 2015) and 2D (Salasnich and Toigo, 2015). We shall discuss the key ideas of these calculations in the next two subsections.

\subsection{Three-dimensional attractive Fermi gas}

Scattering theory plays an essential role in the description of a threedimensional attractive Fermi gas which undergoes BCS-BEC crossover. As recalled above, the bare interaction strength $g$ appearing in the Lagrangian density (118) is related to the physical s-wave scattering length $a_{F}$ of fermions by

$$
\frac{m}{4 \pi \hbar^{2} a_{F}}=\frac{1}{g}+\frac{1}{L^{3}} \sum_{|\mathbf{k}|<\Lambda} \frac{m}{\hbar^{2} k^{2}},
$$

where, as usual, the ultraviolet cutoff $\Lambda$ is introduced to avoid the divergence of the second term on the right side. We recall at this point that 


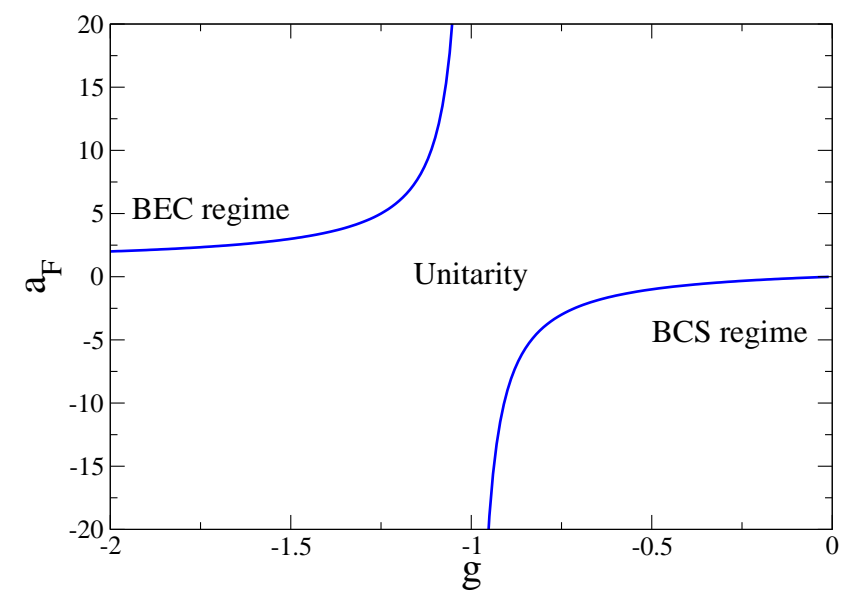

Figure 4: Fermionic scattering length $a_{F}$ as a function of the bare interaction strength $g$, for a finite ultraviolet cutoff $\Lambda$, see Eq. (137). In the plot $a_{F}$ is in units of $m /\left(4 \pi \hbar^{2}\right)$ and $m \Lambda /\left(2 \pi^{2} \hbar^{2}\right)=1$.

the low energy scattering length is negative for an attractive potential if no bound states are present, while it becomes positive when the interaction is so attractive as to admit a bound state. Eq. (136) allows for the change of sign of $a_{F}$ as the strength of the attractive potential becomes more and more negative. In fact, in the continuum limit $\sum_{\mathbf{k}} \rightarrow L^{3} \int d^{3} \mathbf{k} /(2 \pi)^{3}$, after integration over momenta, it reads

$$
\frac{m}{4 \pi \hbar^{2} a_{F}}=\frac{1}{g}+\frac{m}{2 \pi^{2} \hbar^{2}} \Lambda \text {. }
$$

Therefore, in the weak-coupling BCS limit, where $g \rightarrow 0^{-}$, the first term on the r.h.s. of Eq. (137) dominates and $a_{F}=m g /\left(4 \pi \hbar^{2}\right) \rightarrow 0^{-}$, while in the strong-coupling BEC limit, where $g \rightarrow-\infty$, the second term on the r.h.s. of Eq. (137) dominates and $a_{F}=\pi /(2 \Lambda) \rightarrow 0^{+}$when $\Lambda$ is sent to infinity (Gurarie and Radzihovsky, 2007; Schakel, 2008).

In addition, from Eq. (137) one finds that a bound state, that is a pole in the T-matrix of Eq. (42), is possible only if $g<-2 \pi^{2} \hbar^{2} /(m \Lambda)$. See Fig. 4. In the presence of a bound state of energy $-\epsilon_{B}$, for the T-matrix one has

$$
\frac{1}{T\left(-\epsilon_{B}\right)}=0
$$

and consequently from Eq. (41) it follows

$$
-\frac{1}{g}=\frac{1}{L^{3}} \sum_{|\mathbf{k}|<\Lambda} \frac{m}{\hbar^{2} k^{2}+m \epsilon_{B}} .
$$


After integration over momenta one obtains

$$
-\frac{1}{g}=\frac{m}{2 \pi^{2} \hbar^{2}}\left(\Lambda-\sqrt{\frac{m \epsilon_{B}}{\hbar^{2}}} \arctan \left(\frac{\Lambda}{\sqrt{\frac{m \epsilon_{B}}{\hbar^{2}}}}\right)\right),
$$

and comparing Eq. (137) with Eq. (140) one finds

$$
a_{F}=\sqrt{\frac{\hbar^{2}}{m \epsilon_{B}}} \frac{\pi}{2 \arctan \left(\Lambda \sqrt{\frac{\hbar^{2}}{m \epsilon_{B}}}\right)} .
$$

In the limit $\Lambda \rightarrow+\infty$ from this interesting formula one gets

$$
\epsilon_{B}=\frac{\hbar^{2}}{m a_{F}^{2}}
$$

that is the familiar relation between the bound-state energy and the 3D s-wave scattering length $a_{F}$.

In 2015 Salasnich and Bighin (2015) found the regularized zero-temperature grand potential of the fermionic superfluid in the deep BEC regime in the form

$$
\Omega=-L^{3} \frac{(1+\alpha)}{256 \pi}\left(\frac{2 m}{\hbar^{2}}\right)^{3 / 2} \frac{\Delta_{0}^{4}}{|\mu|^{3 / 2}}
$$

with $\alpha=2$ due to zero-point Gaussian fluctuations, performing cutoff regularization and renormalization of Gaussian fluctuations (with a procedure very similar to the one discussed in Section IV.B for three-dimensional interacting bosons), and taking into account the result

$$
\Lambda=\frac{\pi}{2 a_{F}}
$$

from Eq. (137) when $g \rightarrow-\infty$. Let us briefly discuss the derivation of Eq. (143). In the deep BEC regime of the crossover, where the fermionic scattering length $a_{F}$ becomes positive, the chemical potential $\mu$ becomes negative and the regularized zero-temperature mean-field grand potential reads (Salasnich and Bighin, 2015)

$$
\Omega_{m f}=-L^{3} \frac{1}{256 \pi}\left(\frac{2 m}{\hbar^{2}}\right)^{3 / 2} \frac{\Delta_{0}^{4}}{|\mu|^{3 / 2}} .
$$

This expression may be obtained following Leggett seminal paper on the BEC-BCS crossover (Leggett, 1980) by using the convergence-factor regularization supplemented by Eq. (136) and letting $\Lambda \rightarrow \infty$. It may also 
be obtained easily through dimensional regularization or momentum-cutoff regularization, using procedures which are very similar to the ones discussed in previous sections for bosons.

Regularization of the contribution to $\Omega$ from the bosonic Gaussian fluctuations $\Omega_{g}^{(0)}$ is more delicate. In fact, expanding the cutoff-regularized zero-temperature Gaussian grand potential, Eq. (134), in powers of the momentum-cutoff $\Lambda$ one finds at zero temperature (Salasnich and Bighin, 2015)

$$
\begin{aligned}
\frac{\Omega_{g}^{(0)}}{L^{3}} & =\frac{\hbar^{2} \lambda^{1 / 2}}{40 \pi^{2} m} \Lambda^{5}+\frac{m c_{B}^{2}}{12 \pi^{2} \lambda^{1 / 2}} \Lambda^{3} \\
& -\frac{m^{3} c_{B}^{4}}{4 \pi^{2} \lambda^{3 / 2}} \Lambda+\frac{8 m^{4} c_{B}^{5}}{15 \pi^{2} \hbar^{2} \lambda^{2}}+O\left(\frac{1}{\Lambda}\right) .
\end{aligned}
$$

The term proportional to $\Lambda^{5}$ can be neglected since it does not depend on $\mu$ in the deep BEC limit where $\lambda=1 / 4$. In the same limit the term proportional to $\Lambda^{3}$ can be absorbed by renormalizing $\mu$. Notice that the term proportional to $\Lambda$ is finite because in the BEC limit $c_{B}^{4}$ goes to zero faster than $1 / \Lambda$ (Salasnich and Bighin, 2015). The same result is obtained by using the convergence-factor regularization which gives an additional negative energy $-\left(\lambda^{1 / 2} \hbar^{2} q^{2} /(2 m)+m c_{B}^{2} / \lambda^{1 / 2}\right)$ in the bosonic Gaussian grand potential $\Omega_{g}^{(0)}$ of Eq. (134) that exactly cancels the terms proportional to $\Lambda^{5}$ and $\Lambda^{3}$ of Eq. (146).

Remarkably, the term of Eq. (146) which is independent of the momentum cutoff $\Lambda$, i.e

$$
\Omega_{g}^{(\Lambda-\text { independent })}=L^{3} \frac{8 m^{4} c_{B}^{5}}{15 \pi^{2} \hbar^{2} \lambda^{2}},
$$

in the deep BEC regime is subleading with respect to the cutoff-regularized term proportional to $\Lambda$. Thus, in the deep BEC regime, where $|\mu|=$ $\hbar^{2} /\left(2 m a_{F}^{2}\right), \lambda=1 / 4$ and $m c_{B}^{2}=\Delta_{0}^{2} /\left(8|\mu|\right.$ ) (notice that $\lambda$ and $c_{B}$ appear in the collective bosonic excitations of Eq. (117)), the leading convergent term of Eq. (146) is

$$
\Omega_{g}=-L^{3} \frac{\alpha}{256 \pi}\left(\frac{2 m}{\hbar^{2}}\right)^{3 / 2} \frac{\Delta_{0}^{4}}{|\mu|^{3 / 2}},
$$

with $\alpha=2$ (Salasnich and Bighin, 2015). Even more remarkable is the fact that performing dimensional regularization of Eq. (134) one gets directly Eq. (147). In other words, dimensional regularization of Eq. (134) does not give the same result of momentum-cutoff regularization and convergence-factor 
regularization because in our momentum-cutoff (or convergence-factor) regularization $\Lambda$ is constrained by Eq. (144). Clearly, within our momentumcutoff (or convergence-factor) regularization scheme, from Eqs. (145) and (148) one immediately obtains Eq. (143).

In conclusion, in the deep BEC regime the leading term of the zerotemperature one-loop grand potential can be written as

$$
\Omega=\Omega_{m f}+\Omega_{g}=-L^{3}(1+\alpha) \frac{m}{2 \pi \hbar^{2} a_{F}}\left(\mu+\frac{1}{2} \epsilon_{B}\right)^{2},
$$

having taken into account the result (Diener et al., 2008; Salasnich and Bighin, 2015)

$$
\mu=-\frac{1}{2} \epsilon_{B}+\frac{1}{4} \frac{\Delta_{0}^{2}}{\epsilon_{B}}
$$

with $\epsilon_{B}=\hbar^{2} /\left(m a_{F}^{2}\right)$ derived in the BEC regime $\left(a_{F} \rightarrow 0^{+}\right)$from the gap equation

$$
\left(\frac{\partial \Omega_{m f}}{\partial \Delta_{0}}\right)_{L^{3}, \mu}=0
$$

Eq. (149) is the familiar grand potential

$$
\Omega=L^{3} \frac{m_{B}}{8 \pi \hbar^{2} a_{B}} \mu_{B}^{2}
$$

of weakly-interacting repulsive composite bosons of mass $m_{B}=2 m$, density $n_{B}=n / 2$, chemical potential $\mu_{B}=2\left(\mu+\epsilon_{B} / 2\right)$, and the boson-boson scattering length

$$
a_{B}=\frac{2}{(1+\alpha)}=\frac{2}{3} a_{F} .
$$

This result is in good agreement with other beyond-mean-field theoretical predictions: $a_{B} / a_{F} \simeq 0.75$ based on a diagrammatic approach (Pieri and Strinati, 2008), $a_{B} / a_{F} \simeq 0.60$ derived from a four-body analysis (Petrov et al., 2004) and also from Monte Carlo simulations (Astrakharchik et al., 2004), and $a_{B} / a_{F} \simeq 0.55$ obtained with convergence factors ( $\mathrm{Hu}$ et al., 2006; ; Diener et al. , 2008). However, contrary to all other predictions wich are based at some points on numerical calculations, our result, based on a transparent cutoff regularization and subsequent renormalization of bare physical parameters (Salasnich and Bighin, 2015), is fully analytical.

\subsection{Two-dimensional attractive Fermi gas}

In the analysis of the two-dimensional attractive Fermi gas one must remember that, contrary to the three-dimensional case, two-dimensional realistic interatomic potentials for alkali atoms always exhibit a bound state 
and correspondingly a positive two-dimensional s-wave scattering length (Randeria et al., 1990; Marini et al., 1998; Bertaina and Giorgini, 2011). In particular, according to Mora and Castin (Mora and Castin, 2009) the binding energy $\epsilon_{b}>0$ of two fermions can be written in terms of the positive two-dimensional fermionic scattering length $a_{F}$ as

$$
\epsilon_{b}=\frac{4}{e^{2 \gamma}} \frac{\hbar^{2}}{m a_{F}^{2}},
$$

where $\gamma=0.577 \ldots$ is the Euler-Mascheroni constant. Moreover, the attractive (negative) interaction strength $g$ of s-wave pairing is related to the binding energy $\epsilon_{b}>0$ of a fermion pair in vacuum by the expression (Randeria et al., 1989)

$$
-\frac{1}{g}=\frac{1}{2 L^{2}} \sum_{\mathbf{k}} \frac{1}{\frac{\hbar^{2} k^{2}}{2 m}+\frac{1}{2} \epsilon_{b}} .
$$

Randeria et al. (Randeria et al., 1990) showed that in the two-dimensional BCS-BEC crossover, at zero temperature $(T=0)$ the mean-field grand potential $\Omega_{m f}$ can be written as

$$
\Omega_{m f}=-\frac{m L^{2}}{2 \pi \hbar^{2}}\left(\mu+\frac{1}{2} \epsilon_{b}\right)^{2}
$$

taking into account that the zero-temperature 2D gap equation gives (Randeria et al. 1990)

$$
\Delta_{0}=\sqrt{2 \epsilon_{b}\left(\mu+\epsilon_{b} / 2\right)} \text {. }
$$

By using

$$
n=-\frac{1}{L^{2}} \frac{\partial \Omega_{m f}}{\partial \mu}
$$

one immediately finds the chemical potential $\mu$ as a function of the number density $n=N / L^{2}$, i.e.

$$
\mu=\frac{\pi \hbar^{2}}{m} n-\frac{1}{2} \epsilon_{b}
$$

In the BCS regime, where $\epsilon_{b} \ll \epsilon_{F}$, with $\epsilon_{F}=\pi \hbar^{2} n / m$, one finds $\mu \simeq \epsilon_{F}>0$ while in the BEC regime, where $\epsilon_{b} \gg \epsilon_{F}$ one has $\mu \simeq-\epsilon_{b} / 2<0$. Clearly, both Eqs. (156) and (159) do not reproduce the expected logarithmic behavior in the deep BEC regime, where there should be a two-dimensional Bose gas of repulsive composite bosons (Schick, 1971; Popov, 1972). 
By performing dimensional regularization of Gaussian fluctuations (with a procedure that is very similar to the one discussed in Section III.C for twodimensional interacting bosons), we have recently found (Salasnich and Toigo, 2015) that the zero-temperature grand potential becomes

$$
\Omega=-\frac{m L^{2}}{64 \pi \hbar^{2}}\left(\mu+\frac{1}{2} \epsilon_{b}\right)^{2} \ln \left(\frac{\epsilon_{b}}{2\left(\mu+\frac{1}{2} \epsilon_{b}\right)}\right),
$$

in the deep BEC regime of this two-dimensional Fermi superfluid, where the chemical potential $\mu$ becomes negative and $\lambda$ of Eq. (117) goes to $1 / 4$.

Let us briefly discuss the derivation of Eq. (160). Setting $g_{0}=\pi \hbar^{2} / m$ and $\mu_{0}=\mu+\epsilon_{b} / 2$, the $2 \mathrm{D}$ zero-temperature mean-field grand potential (156) can be written as

$$
\Omega_{m f}=-L^{2} \frac{\mu_{0}^{2}}{2 g_{0}} .
$$

In addition, the Bogoliubov's sped of sound $c_{B}$ which appears in Eq. (117) satisfies the relation (Marini et al., 1998; Salasnich and Toigo, 2015)

$$
m c_{B}^{2}=\mu+\frac{1}{2} \epsilon_{b}=\mu_{0},
$$

and consequently the 2D zero-temperature Gaussian grand potential becomes

$$
\Omega_{g}=L^{2} \int \frac{d^{2} \mathbf{q}}{(2 \pi)^{2}} \sqrt{\frac{\hbar^{2} q^{2}}{2 m}\left(\lambda \frac{\hbar^{2} q^{2}}{2 m}+2 \mu_{0}\right)}
$$

with $\lambda=1 / 4$ in the deep BEC regime (Salasnich and Toigo, 2015). Quite remarkably, the two-dimensional Eqs. (161) and (163) for the attractive Fermi gas are formally equivalent to Eqs. (28) and (39) of the $D$-dimensional repulsive Bose gas when $D=2$ (apart for the value of $\lambda$ that is equal to one for repulsive bosons). Thus, one can use one of the three regularization procedures discussed in the previous sections to get the zero-temperature regularized total grand potential in the deep BEC regime (where $\lambda=1 / 4$ ), that is Eq. (160). In our recent paper (Salasnich and Toigo, 2015) we have used dimensional regularization, which has the advantage of being independent of scattering theory.

Introducing $\mu_{B}=2\left(\mu+\epsilon_{b} / 2\right)$ as the chemical potential of composite bosons with mass $m_{B}=2 m$ and density $n_{B}=n / 2$, the zero-temperature total grand potential (160) can be rewritten as

$$
\Omega=-\frac{m_{B} L^{2}}{8 \pi \hbar^{2}} \mu_{B}^{2} \ln \left(\frac{\epsilon_{b}}{\mu_{B}}\right)
$$


As usual, the total density of bosons $n_{B}=n / 2$ is obtained in terms of $\mu_{B}=2\left(\mu+\epsilon_{b} / 2\right)$ from the zero-temperature thermodynamic formula

$$
n=-\frac{1}{L^{2}} \frac{\partial \Omega}{\partial \mu}
$$

which leads to:

$$
n_{B}=\frac{m_{B}}{4 \pi \hbar^{2}} \mu_{B} \ln \left(\frac{\epsilon_{b}}{\mu_{B} e^{1 / 2}}\right) .
$$

Inserting Eq. (154), which gives the binding energy $\epsilon_{b}$ of two fermions in terms of their s-wave scattering length $a_{F}$, into Eq. (166) we exactly recover Popov's 2D equation of state (Popov, 1972) of weakly-interacting bosons with scattering length $a_{B}$, i.e.

$$
n_{B}=\frac{m_{B}}{4 \pi \hbar^{2}} \mu_{B} \ln \left(\frac{4 \hbar^{2}}{m_{B} \mu_{B} a_{B}^{2} e^{2 \gamma+1}}\right),
$$

provided that we identify the effective bosonic scattering length $a_{B}$ with (Salasnich and Toigo, 2015):

$$
a_{B}=\frac{1}{2^{1 / 2} e^{1 / 4}} a_{F} .
$$

Remarkably, the value $a_{B} / a_{F}=1 /\left(2^{1 / 2} e^{1 / 4}\right) \simeq 0.551$ from this analytical formula is in full agreement with $a_{B} / a_{F}=0.55(4)$ obtained by Monte Carlo calculations (Bertaina and Giorgini, 2011; Bertaina, 2013) and $a_{B} / a_{F}=$ 0.56 very recently derived by using Gaussian fluctuations with convergencefactor regularization ( $\mathrm{He}$ et al., 2015).

\subsection{One-dimensional attractive Fermi gas}

Even if we know that the Gaussian approximation is inadequate to treat strongly interacting Bose systems in 1D (Lieb and Liniger, 1963), nonetheless for the sake of completeness in this subsection we analyze the onedimensional $(D=1)$ attractive Fermi gas at zero temperature taking into account the mean-field contributions of Eqs. (130) and (131) due to fermionic single-particle excitations (116) and the Gaussian quantum fluctuations of Eq. (134), due to bosonic collective excitations (117). The 1D problem of fermions with contact attractive interaction was exactly solved in 1967 by Gaudin using the Bethe ansatz (Gaudin, 1967). Similarly to the twodimensional case, also for the 1D attractive Fermi gas for any strength $g<0$ it exists a bound state of energy $\epsilon_{b}$. The chemical potential $\mu$ is positive 
in the BCS regime of weak attraction while it becomes negative and approaches $-\epsilon_{b} / 2$ in the Tonks-like regime of strong attraction (Gaudin, 1967; Fuchs et al., 2004). We use the words "Tonks-like" because, as we shall see, this strongly-attractive regime of $1 \mathrm{D}$ fermions is actually a Tonks-Girardeau regime (Girardeau, 1960), where there is no quasi-BEC but instead there is a gas of strongly-repulsive 1D bosons (Gaudin, 1967; Fuchs et al., 2004).

More than twenty years ago Casas et al. (Casas et al., 1991) have studied the zero-temperature 1D mean-field theory. ¿From their results one immediately finds that in the deep Tonks-like regime $\left(\left(\mu+\epsilon_{b} / 2\right) / \epsilon_{b} / 2 \ll 1\right)$ Eqs. (130) and (131) give

$$
\Omega_{m f}=-\frac{L}{2 \epsilon_{b}^{1 / 2}}\left(\frac{m}{\hbar^{2}}\right)^{1 / 2}\left(\mu+\frac{1}{2} \epsilon_{b}\right)^{2}
$$

where $\epsilon_{B}=m g^{2} /\left(4 \hbar^{2}\right)$ is the binding energy of fermionic pairs (Casas et al., 1991). Setting $g_{0}=-g=2\left(\hbar^{2} \epsilon_{b} / m\right)^{1 / 2}$ and $\mu_{0}=\mu+\epsilon_{b} / 2$, the $1 \mathrm{D}$ zerotemperature mean-field grand potential can be rewritten as

$$
\Omega_{m f}=-L \frac{\mu_{0}^{2}}{2 g_{0}} .
$$

In addition, also in 1D the Bogoliubov's speed of sound $c_{B}$ which appears in Eq. (117) satisfies the formula (162) and consequently the $1 \mathrm{D}$ zerotemperature Gaussian grand potential becomes

$$
\Omega_{g}=L \int_{-\infty}^{+\infty} \frac{d q}{(2 \pi)} \sqrt{\frac{\hbar^{2} q^{2}}{2 m}\left(\lambda \frac{\hbar^{2} q^{2}}{2 m}+2 \mu_{0}\right)}
$$

again with $\lambda=1 / 4$ in the deep Tonks-like regime. Clearly, Eqs. (170) and (171) of the attractive 1D Fermi gas are formally equivalent to Eqs. (28) and (39) of the $D$-dimensional repulsive Bose gas when $D=1$ (apart for $\lambda$, that is equal to one for repulsive bosons). Thus, we can again use one of the three regularization procedures discussed in the previous sections to get the zero-temperature regularized total grand potential $\Omega=\Omega_{m f}+\Omega_{g}$ in the deep Tonks-like regime (where $\lambda=1 / 4$ ). In this way we find

$$
\frac{\Omega}{L}=-\left(\frac{m}{\hbar^{2}}\right)^{1 / 2}\left[\frac{1}{2 \epsilon_{b}^{1 / 2}}\left(\mu+\frac{1}{2} \epsilon_{b}\right)^{2}+\frac{8}{3 \pi}\left(\mu+\frac{1}{2} \epsilon_{b}\right)^{3 / 2}\right],
$$

showing that in the deep Tonks-like regime the mean-field contribution to the zero-temperature grand potential is subleading with respect to the Gaussian 
one. Using the thermodynamic relation

$$
n=-\frac{1}{L} \frac{\partial \Omega}{\partial \mu}
$$

for the $1 \mathrm{D}$ number density $n=N / L$ of fermions and the leading Gaussian term for the grand potential we find

$$
\mu=-\frac{1}{2} \epsilon_{b}+\frac{\pi^{2} \hbar^{2}}{16 m} n^{2}
$$

in the deep Tonks-like regime of strong interaction, which corresponds to the very dilute limit. The term $-\epsilon_{b} / 2$ of Eq. (174) is exactly the first term of Gaudin theory (Gaudin, 1967) in a low-density series expansion in powers of $n$ (Casas et al., 1991). The term $\pi^{2} \hbar^{2} n^{2} /(16 m)$ of Eq. (174) is similar but not equal to the second term of the exact Gaudin expansion, that instead gives $\pi^{2} \hbar^{2} n^{2} /(32 m)$ (Casas et al., 1991). Thus, in the one dimensional case Gaussian fluctuations improve the mean-field theory but do not produce the correct equation of state in the Tonks-like regime. We remind that a Tonks gas can also be obtained starting from a 1D repulsive Bose gas by strongly increasing its positive interaction strength (Lieb and Liniger, 1963).

\section{Experiments vs theory for fermionic superfluids}

\subsection{Attractive Fermi gas in $3 D$}

Beyond-mean-field effects in the frequencies of collective excitations of a 3D fermionic superfluid under external confinement were first predicted by Stringari (2004) and experimentally detected in a dilute gas of ${ }^{6} \mathrm{Li}$ atoms at very low temperatures by Bartenstein et al. (2004) and by Altmever et al. (2007). In these experiments the atomic gas was confined by an external anisotropic harmonic potential

$$
U(\mathbf{r})=\frac{m}{2}\left(\omega_{\perp}^{2}\left(x^{2}+y^{2}\right)+\omega_{z}^{2} z^{2}\right),
$$

where $\omega_{\perp}$ and $\omega_{z}$ are the cylindric radial and longitudinal frequencies, respectively. The collective dynamics of the system is described efficiently by the hydrodynamic equations of superfluids (for a review see Giorgini. Pitaevskii, and Stringari $(2008))$, modified by the inclusion of the external potential $U(\mathbf{r})$, namely

$$
\begin{aligned}
\frac{\partial n}{\partial t}+\nabla \cdot(n \mathbf{v}) & =0 \\
m \frac{\partial \mathbf{v}}{\partial t}+\nabla\left[\frac{1}{2} m v^{2}+\mu\left[n, a_{F}\right]+U(\mathbf{r})\right] & =\mathbf{0} .
\end{aligned}
$$


where the zero-temperature equation of state is encoded in the explicit expression of the chemical potential $\mu$ as a function of the local density $n$ and of the s-wave scattering length $a_{F}$ of fermions. For a 3D system of attractive fermions one may find analytical solutions of Eqs. (176) and (177) corresponding to the breathing collective modes, both in the BEC $\left(a_{F} \rightarrow 0^{+}\right)$and $\operatorname{BCS}\left(a_{F} \rightarrow 0^{-}\right)$regimes and at unitarity $\left(\left|a_{F}\right| \rightarrow \infty\right)$ (Stringari (2004)). As a matter of fact analytic expressions for the collective frequencies (see for instance Giorgini, Pitaevskii, and Stringari (2008)) may also be calculated if the equation of state is of the type $\mu=\mu_{0} n^{\gamma}$ for (polytropic equation of state). For very elongated cigar-shaped traps $\left(\omega_{\perp} / \omega_{z} \gg 1\right)$ the collective radial breathing mode frequency $\Omega_{\perp}$ is given by

$$
\Omega_{\perp}=\sqrt{2(\gamma+1)} \omega_{\perp},
$$

while the collective longitudinal breathing mode $\Omega_{z}$ is

$$
\Omega_{z}=\sqrt{\frac{3 \gamma+2}{\gamma+1}} \omega_{z}
$$

Manini and Salasnich (2005) interpolated between the BEC (with Lee,Huang,Yang (Lee, Huang and Yang (1957)) correction and BCS (with mean field interaction) through the unitary regime, by introducing an effective polytropic index $\gamma$ as the logarithmic derivative of the chemical potential $\mu$, that is

$$
\gamma=\frac{n}{\mu} \frac{\partial \mu}{\partial n}
$$

This approach (Manini and Salasnich (2005)) predicted relevant deviations from the mean-field results for the frequencies of collective breathing modes of a two-component Fermi gas of ${ }^{6} \mathrm{Li}$ atoms to unitarity $\left(a_{F}=\infty\right)$, which were confirmed by the experiment of Altmeyer et al. (2007).

A direct measurement of the equation of state of an attractive ultracold fermions system was performed by Navon et al. (2010) by absorption imaging an harmonically trapped sample of ${ }^{6} \mathrm{Li}$ atoms at ultralow temperatures. Parametrizing their data for the pressure vs. density with analytical functions Navon et al. (2010) were able to extract relevant physical quantities, such as beyond mean-field corrections, for the superfluid system in the entire BCS-BEC crossover. In particular, in the BEC regime, the data are well reproduced by the equation of state of superfluid dilute composite bosons (Levronas and Combescot (2007)) confirming the coefficient of the Lee, Huang and Yang (1957) term and allowed the first experimental determination of the scattering length between composite bosons in terms of scattering length between fermions as $a_{B}=0.6 a_{F}$. 


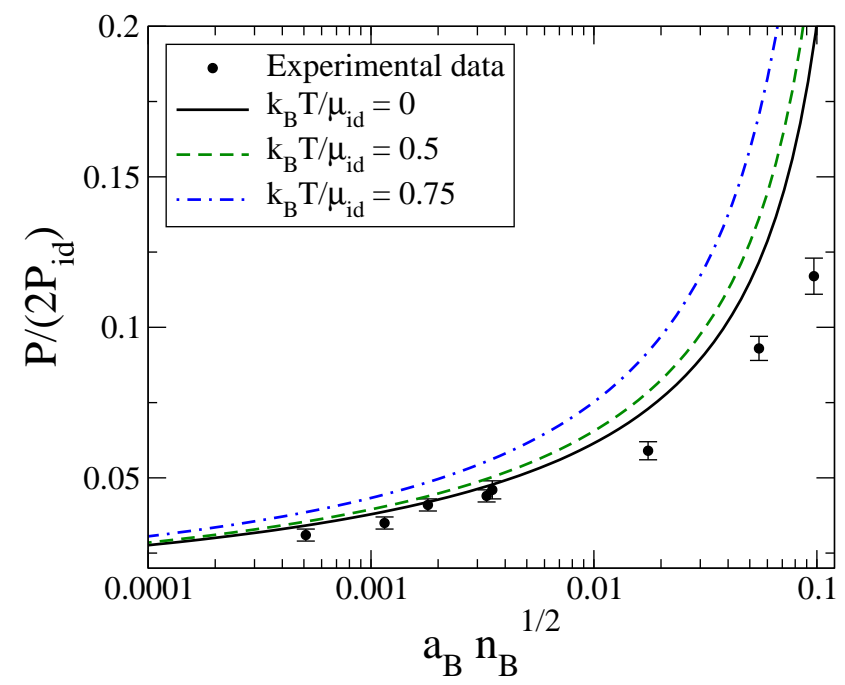

Figure 5: Scaled pressure $P /\left(2 P_{i d}\right)$ of the $2 \mathrm{D}$ gas of composite bosons as a function of the bosonic gas parameter $a_{B} n_{B}^{1 / 2}$, where $P_{i d}=2 \pi \hbar^{2} n_{B}^{2} / m_{B}$ is the pressure of an ideal $2 \mathrm{D}$ gas with $m_{B}$ the mass of each bosonic particle, $a_{B}$ is the s-wave scattering length of bosons, and $n_{B}$ is the bosonic $2 \mathrm{D}$ density. The filled circles with error bars are the experimental data of Makhalov et al. (2014). The curves are obtained from Eqs. (154), (168) (184) and (185) for different values of the scaled temperature $k_{b} T / \mu_{i d}$, with $\mu_{i d}=4 \pi \hbar^{2} n_{B} / m_{B}$. Notice that the BCS-BEC mean-field theory in the BEC regime predicts a constant pressure, independent of the scattering length.

\subsection{Attractive Fermi gas in $2 D$}

Recently Makhalov, Martiyanov, and Turlapov (2014) have realized a quasi-2D Fermi system with widely tunable s-wave interactions nearly in a ground state, investigating an ultracold gas of atoms by measuring the pressure $P$ as a function of the density $n$. The experiment of Makhalov et al. (2014) covers physically different regimes corresponding to weakly or strongly attractive Fermi gases or a Bose gas of tightly bound pairs of fermions.

Within our one-loop Gaussian approach, the pressure $P$ is immediately obtained from Eqs. (135) and (164) using the thermodynamic relation $\Omega=$ $-P L^{2}$ :

$$
\begin{aligned}
P & =\frac{m_{B}}{8 \pi \hbar^{2}} \ln \left(\frac{\epsilon_{b}}{\mu_{B}}\right) \mu_{B}^{2} \\
& -\frac{1}{\beta L^{2}} \sum_{\mathbf{q}} \ln \left(1-e^{-\beta E_{c o l}(q)}\right),
\end{aligned}
$$


because, in practice, the gapped single-particle fermionic excitations $E_{s p}(k)$ do not contribute to thermal properties since $\beta \Delta_{0}$ is extremely large in the $\mathrm{BEC}$ regime. Morever, the density $n_{B}$ is given by

$$
n_{B}=\left(\frac{\partial P}{\partial \mu_{B}}\right)_{T, L^{2}}
$$

from which one finds

$$
\begin{aligned}
n_{B} & =\frac{m_{B}}{4 \pi \hbar^{2}} \ln \left(\frac{\epsilon_{b}}{\mu_{B} e^{1 / 2}}\right) \mu_{B} \\
& -\frac{1}{\beta L^{2}} \sum_{\mathbf{q}} \frac{\partial E_{c o l}(q)}{\partial \mu_{B}} \frac{1}{e^{\beta E_{c o l}(q)}-1} .
\end{aligned}
$$

We now use $E_{\text {col }}(q) \simeq\left(\mu_{B} / m_{B}\right)^{1 / 2} \hbar q$ and the continuum limit $\sum_{\mathbf{q}} \rightarrow$ $L^{2} \int d^{2} \mathbf{q} /(2 \pi)^{2}$. In this way we get

$$
n_{B}=\frac{m_{B}}{4 \pi \hbar^{2}} \mu_{B}\left[\ln \left(\frac{\epsilon_{b}}{\mu_{B} e^{1 / 2}}\right)-2 \zeta(3)\left(\frac{k_{B} T}{\mu_{B}}\right)^{3}\right]
$$

and clearly also

$$
P=\frac{m_{B}}{8 \pi \hbar^{2}} \mu_{B}^{2}\left[\ln \left(\frac{\epsilon_{b}}{\mu_{B}}\right)+4 \zeta(3)\left(\frac{k_{B} T}{\mu_{B}}\right)^{3}\right],
$$

where $\zeta(x)$ is the Riemann zeta fuction and $\zeta(3)=1.20205$. Eqs. (184) and (185) give, at fixed $k_{B} T / \mu_{B}$, a parametric formula for the the pressure $P$ as a function of the density $n_{B}$ where $\mu_{B}$ is the dummy parameter.

Taking into account Eqs. (154) and (168), in Fig. 5 we plot the pressure $P$ in units of the ideal pressure $P_{i d}=2 \pi \hbar^{2} n_{B}^{2} / m_{B}$ as a function of the adimensional gas parameter $a_{B} n_{B}^{1 / 2}$. In the deep weak-coupling regime $a_{B} n_{B}^{1 / 2}<0.01$ and at very low temperature $k_{B} T / \mu_{i d} \ll 1$ with $\mu_{i d}=4 \pi \hbar^{2} n_{B} / m_{B}$, the figure shows a good agreement between the experimental data of Makhalov et al. (2014) and our theoretical curves. Actually the figure suggests that the atomic cloud of Makhalov et al. (2014) was practically at zero temperature. The deviations between theory and experiments at larger values of the gas parameter $a_{B} n_{B}^{1 / 2}$ are presumably due to incomplete bosonization of fermionic pairs.

It is important to stress that the bosonic collective excitations $E_{c o l}(q)$ are given by Eq. (117) only in the deep BEC regime. In the full BCS-BEC 
crossover, $E_{\text {col }}(q)$ can be obtained numerically setting $\operatorname{det}(\mathbf{M}(Q))=0$, where $\mathbf{M}(Q)$ is the inverse propagator for the pair fluctuations, which appears in the Gaussian action (see Diener et al. (2008))

$$
S_{g}\left[\eta, \bar{\eta}, \Delta_{0}\right]=\frac{1}{2} \sum_{Q}(\bar{\eta}(Q), \eta(-Q)) \mathbf{M}(Q)\left(\begin{array}{c}
\eta(Q) \\
\bar{\eta}(-Q)
\end{array}\right)
$$

having introduced the Fourier-transformed version of the fluctuation fields, with $Q=\left(\mathrm{i} \Omega_{n}, \mathbf{q}\right), \Omega_{n}=2 \pi n / \beta$ being the Bose Matsubara frequencies. The matrix elements of $\mathbf{M}(Q)$ are defined by

$$
\begin{gathered}
\mathbb{M}_{11}(Q)=\frac{1}{g}+\sum_{\mathbf{k}}\left(\frac{u^{2} u^{\prime 2}}{\mathrm{i} \omega_{n}-E-E^{\prime}}-\frac{v^{2} v^{\prime 2}}{\mathrm{i} \omega_{n}+E+E^{\prime}}\right) \\
\mathbb{M}_{12}(Q)=\sum_{\mathbf{k}} u v u^{\prime} v^{\prime}\left(\frac{1}{\mathrm{i} \omega_{n}+E+E^{\prime}}-\frac{1}{\mathrm{i} \omega_{n}-E-E^{\prime}}\right)
\end{gathered}
$$

where $u=u_{\mathbf{k}}=\sqrt{\frac{1}{2}\left(1+\frac{\epsilon_{\mathbf{k}}-\mu}{E_{s p}(\mathbf{k})}\right)}, v=v_{\mathbf{k}}=\sqrt{1-u_{\mathbf{k}}^{2}}, u^{\prime}=u_{\mathbf{k}+\mathbf{q}}, v^{\prime}=v_{\mathbf{k}+\mathbf{q}}$, $E=E_{s p}(\mathbf{k}), E^{\prime}=E_{s p}(\mathbf{k}+\mathbf{q})$. The remaining matrix elements are defined by the relations: $\mathbb{M}_{22}(q)=\mathbb{M}_{11}(-q), \mathbb{M}_{21}(q)=\mathbb{M}_{12}(q)$.

Quite remarkably, He et al. (2015) have numerically found a very good agreement between the experimental data of Makhalov et al. (2014) and the one-loop Gaussian theory in the full BCS-BEC crossover by using the zero-temperature grand potential

$$
\Omega_{g}(\mu, T)=\frac{1}{2 \beta} \sum_{Q} \ln \left[\frac{\mathbb{M}_{11}(Q)}{\mathbb{M}_{22}(Q)} \operatorname{det}(\mathbf{M}(Q))\right] e^{\mathrm{i} \Omega_{n} 0^{+}},
$$

which is regularized by convergence factors (Diener et al. (2008); He et al. (2015)). As previously discussed, in the BEC regime of composite bosons He et al. (2015) have recovered our analytical result, Eq. (154). In a very recent paper Bighin and Salasnich (2016) have compared the first sound velocity $c_{s}$ of the $2 \mathrm{D}$ Fermi superfluid with preliminar experimental data of Luick (2014), taking into account Eq. (189) and the zero-temperature thermodynamic relation

$$
c_{s}=\sqrt{\frac{n}{m} \frac{\partial \mu}{\partial n}}=\sqrt{-\frac{n}{m}\left(\frac{1}{L^{2}} \frac{\partial^{2} \Omega(\mu)}{\partial \mu^{2}}\right)^{-1}} .
$$


At the mean-field level one has $c_{s}\left(\mu_{m f}\right)=v_{F} / \sqrt{2}$ across the whole BCSBEC crossover, $v_{F}$ being the Fermi velocity. The inclusion of Gaussian fluctuations gives a quite different sound velocity: it slowly tends to the aforementioned value in the BCS limit, showing, on the other hand, a remarkable difference in the intermediate and BEC regimes. The preliminar experimental data of Luick (2014) are in very good agreement with the Gaussian theory of Bighin and Salasnich (2016).

We can conclude that the one-loop Gaussian theory of fermionic superfluids shows good agreement with very recent experimental data (Makhalov et al. (2014); Luick (2014), and also Boetcher et al. (2016)) in the full 2D BCSBEC crossover when collective bosonic excitations are appropriately taken into account. Moreover, in the BEC regime of the crossover the Gaussian theory becomes analytically tractable and, as expected, it gives the one-loop equation of state of $2 \mathrm{D}$ composite bosons whose interaction is characterized by an s-wave scattering length nontrivially related to the scattering length of the atomic fermions.

\subsection{Attractive Fermi gas in $1 D$}

The observation of pairing phenomena in a 1D Fermi gas of ${ }^{40} \mathrm{~K}$ atoms was reported by Moritz et al. (2005). Using radio-frequency spectroscopy they measured the binding energy of two-particle bound states of atoms confined in a one-dimensional matter waveguide. More recently, Liao et al. (2010) measured density profiles of 1D trapped two-spin-component fermionic ${ }^{6} \mathrm{Li}$ atoms. In particular, Liao et al. (2010) analyzed the effect of spin imbalance in the 1D trapped gas finding a partially polarized core surrounded by wings which, depending on the degree of polarization, are composed of either a completely paired or a fully polarized Fermi gas. This kind of phase separation confirms the key features of the phase diagram predicted from the exact Bethe-ansatz solution of the 1D unbalanced uniform Fermi gas (Orso (2007)). As previously stressed, for 1D systems (both bosonic and fermionic) the one-loop Gaussian theory is fully reliable only in the weakcoupling regime.

\section{Conclusions}

We have shown that the zero-point energy of both bosonic and fermionic ultracold atoms contains a finite contribution which plays a relevant role in the determination of a reliable equation of state. In the case of repulsive bosonic atoms the final convergent equation of state, which depends on the dimensionality of the system, is independent of the regularization 
procedure. On the contrary, we have found that the dimensional regularization cannot be used for three-dimensional attractive fermionic atoms which exhibit a BCS-BEC crossover. In fact, the sign of the scattering length whose change from negative to positive across the crossover may be accounted for by using a cutoff regularization, would remain always negative under dimensional regularization. However, the dimensional regularization can be used for the study of the two-dimensional BCS-BEC crossover because in the two-dimensional problem the scattering length does not change sign. By using momentum-cutoff regularization in the three-dimensional case (Salasnich and Bighin, 2015) and dimensional regularization in the twodimensional case (Salasnich and Toigo, 2015) one derives meaningful equations of state in the BEC regime of composite bosons. Quite remarkably, from these equations of state one obtains simple analytical formulas between the scattering length of composite molecular bosons and the scattering length of atomic fermions (Salasnich and Bighin, 2015; Salasnich and Toigo, 2015). Finally, for the one-dimensional Fermi superfluid we have found that Gaussian fluctuations improve the mean-field theory but do not give the correct equation of state in the Tonks-like regime of impenetrable bosons.

There are several open problems for the physics of ultracold atoms which can be faced employing the regularization techniques of Gaussian fluctuations discussed in this paper. In the two-dimensional case (for both bosonic and fermionic superfluids) the Berezinsky-Kosterlitz-Thouless critical temperature (Berezinskii, 1971; Kosterlitz and Thouless, 1973) of the superfluidnormal phase transition can be extracted by using the Thouless criterion (Nagaosa, 1999) and an accurate description of the superfluid density which takes into account Gaussian fluctuations in the finite-temperature equation of state. Gaussian contributions to the equation of state are clearly relevant for Bose-Fermi mixtures (Nishida and Son, 2006) and for unbalanced superfluid fermions (Klimin et al., 2012). For superfluid fermionic atoms in three and two dimensions one can also investigate the effects of Gaussian fluctuations on the zero-temperature condensate fraction (Fukushima et al., 2007) comparing with mean-field results (Salasnich et al., 2005; Salasnich, 2007) and available Monte Carlo calculations (Astrakharchik et al., 2005). In conclusion, we stress that in addition to ultracold atomic gases, there are several other superfluid quantum many-body systems where the methods of functional integration and regularization of Gaussian fluctuations can play a relevant role to achieve a meaningful and reliable theoretical description. Among them we quote high- $\mathrm{T}_{c}$ superconductors (Scalapino, 2012), polar molecules in bilayers (Zinner et al., 2012), neutron matter in the BCSBEC crossover (Salasnich, 2011), quark-gluon plasma (Bhattacharya et al., 
2014), quark matter in stars (Anglani et al., 2014), exciton-polariton condensates (Bvrnes et al., 2014) and, more generally, quantum fluids of light (Carusotto and Ciuti, 2013).

\section{Acknowledgments}

The authors acknowledge Ministero Istruzione Universita Ricerca (PRIN project 2010LLKJBX) for partial support. The authors thank Lara Benfatto, Giacomo Bighin, Massimo Capone, Milton Cole, Luca Dell'Anna, Sergei Klimin, Pieralberto Marchetti, Andrea Perali, Pierbiagio Pieri, Carlos Sa de Melo, Adriaan Schakel, Giancarlo Strinati, Jacques Tempere, and Andrea Trombettoni for enlightening discussions.

\section{References}

\section{References}

Altmeyer, A., S. Riedl, C. Kohstall, M.J. Wright, R. Geursen, M. Bartenstein, C. Chin, J. Hecker Denschlag, and R. Grimm,, 2007, Precision measurements of collective oscillations in the BEC-BS crossover, Phys. Rev. Lett. 98, 040401.

Andersen, J.O. and H. Haugerud, 2002, Ground state of a trapped BoseEinstein condensate in two dimensions: Beyond the mean-field approximation, Phys. Rev. A 65, 033615.

Andersen, J.O., 2004, Theory of the weakly interacting Bose gas, Rev. Mod. Phys. 76, 599 .

Anderson, M.H., J.R. Ensher, M.R. Matthews, C. Wieman, and E.A. Cornell 1995, Observation of Bose-Einstein Condensation in a Dilute Atomic Vapor, Science 269, 198.

Anglani, R., R. Casalbuoni, M. Ciminale, R. Gatto, N. Ippolito, M. Mannarelli, and Marco Ruggieri 2014, Crystalline color superconductors, Rev. Mod. Phys. 86, 509.

Astrakharchik, G.E., J. Boronat, J. Casulleras, and S. Giorgini, 2004, Equation of State of a Fermi Gas in the BEC-BCS Crossover: A Quantum Monte Carlo Study, Phys. Rev. Lett. 93, 200404. 
Astrakharchik, G.E., J. Boronat, J. Casulleras, and S. Giorgini, 2005, Momentum Distribution and Condensate Fraction of a Fermion Gas in the BCS-BEC Crossover, Phys. Rev. Lett. 95, 230405.

Astrakharchik, G.E., J. Boronat, J. Casulleras, I.L. Kurbakov, and Yu.E. Lozovik, 2009, Equation of state of a weakly interacting two-dimensional Bose gas studied at zero temperature by means of quantum Monte Carlo methods, Phys. Rev. A 79, 051602(R).

Altland, A. and B. Simons, 2010, Condensed Matter Field Theory (Cambridge Univ. Press, Cambridge).

Bartenstein, M, A. Altmeyer, S. Riedl, S. Jochim, C. Chin, J. Hecker Denschlag, and R. Grimm,, 2004, Collective excitations of a degenerate gas at the BEC-BCS crossover, Phys. Rev. Lett. 92, 203201.

Bhattacharya, T., M.I. Buchoff, N.H. Christ, H.-T. Ding, R. Gupta, C. Jung, F. Karsch, Z. Lin, R.D. Mawhinney, G. McGlynn, S. Mukherjee, D. Murphy, P. Petreczky, D. Renfrew, C. Schroeder, R.A. Soltz, P.M. Vranas, and H. Yin (HotQCD Collaboration), 2014, QCD Phase Transition with Chiral Quarks and Physical Quark Masses, Phys. Rev. Lett. 113, 082001.

Berezinskii, V.L., 1971, Destruction of Long-range Order in One-dimensional and Two-dimensional Systems having a Continuous Symmetry, Sov. Phys. JETP 32 (3), 493.

Bertaina G. and S Giorgini, 2011, BCS-BEC Crossover in a TwoDimensional Fermi Gas, Phys. Rev. Lett. 106, 110403.

Bertaina, G., 2013, Two-dimensional short-range interacting attractive and repulsive Fermi gases at zero temperature, Eur. Phys. J. Special Topics 217, 153 .

Bighin, G. and L Salasnich, 2016, Finite-temperature quantum fluctuations in two-dimensional Fermi superfluids, Phys. Rev. B 93, 014519.

Bloch, I., J. Dalibard, and W. Zwerger, 2008, Many-body physics with ultracold gases, Rev. Mod. Phys. 80, 885.

Boettcher, I., L. Bayha, D. Kedar, P. A. Murthy, M. Neidig, M. G. Ries, A. N. Wenz, G. Zrn, S. Jochim, and T. Enss, 2016, Equation of State of Ultracold Fermions in the 2D BEC-BCS Crossover Region, Phys. Rev. Lett. 116, 045303. 
Bogoliubov, N.N., 1947, On the theory of superfluidity, J. Phys. (Moscow) 11, 23.

Byrnes, T., N.Y. Kim, and Y. Yamamoto, 2014, Exciton-polariton condensates, Nature Physics 10, 803 (2014).

Bose, S.N., 1924, Planck's Law and Light Quantum Hypothesis, Z. Phys. 26, 178.

Braaten, E. and A. Nieto, 1997, Renormalization effects in a dilute Bose gas, Phys. Rev. B 55, 8090.

Bradley, C.C., C.A. Sackett, J.J. Tollett, and R.G. Hulet, 1995, Evidence of Bose-Einstein Condensation in an Atomic Gas with Attractive Interactions, Phys. Rev. Lett. 75, 1687.

Carussotto, I., and C. Ciuti, 2013, Quantum fluids of light, Rev. Mod. Phys. 85, 299.

Casas, M., C. Esebbag, A. Extremera, J.M. Getino, M. de Llano, A. Plastino, and H. Rubio, 1991, Cooper pairing in a soluble one-dimensional many-fermion model, Phys. Rev. A 44, 4915.

Chien, C-C., J-H. She, and F. Cooper, 2014, Mean-Field Description of Pairing Effects, BKT Physics, and Superfluidity in 2D Bose Gases, Ann. Phys. 347, 192.

Chin, C., et al., 2004, Observation of the Pairing Gap in a Strongly Interacting Fermi Gas, Science 305, 1128.

Coleman, S., 1973, There are no Goldstone bosons in two dimensions, Commun. Math. Phys. 31, 259.

Dalfovo, F., S. Giorgini, L.P. Pitaevskii, and S. Stringari, 1999, Theory of Bose-Einstein condensation in trapped gases, Rev. Mod. Phys. 71, 463.

Davis, K.B., M.O. Mewes, M.R. Andrews, N.J. van Druten, D.S. Durfee, D.M. Kurn, and W. Ketterle, 1995, Bose-Einstein Condensation in a Gas of Sodium Atoms, Phys. Rev. Lett. 75, 3969.

Diener, R.B., R. Sensarma, and M. Randeria, 2008, Quantum fluctuations in the superfluid state of the BCS-BEC crossover, Phys. Rev. A 77, 023626. 
Eagles, D.M., 1969, Possible Pairing without Superconductivity at Low Carrier Concentrations in Bulk and Thin-Film Superconducting Semiconductors, Phys. Rev. 186, 456.

Einstein, A., 1924, Quantentheorie des einatomigen idealen Gases, Sitz. Ber. Preussischen Akad. Wiss. Phys. Math. Kl. 261, 3.

Feynman, R.P., 1948, Relativistic Cut-Off for Quantum Electrodynamics, Phys. Rev. 74, 1430.

Fuchs, J.N., A. Recati, and W. Zwerger, 2004, Exactly Solvable Model of the BCS-BEC Crossover, Phys. Rev. Lett. 93, 090408.

Fukushima, N., Y. Ohashi, E. Taylor, and A. Griffin, 2007, Superfluid density and condensate fraction in the BCS-BEC crossover regime at finite temperatures, Phys. Rev. A 75, 033609.

Gaudin, M., 1967, Un systeme a une dimension de fermions en interaction, Phys. Lett. 24A, 55.

Giorgini, S., P. Pitaevskii, and S. Stringari, 2008, Theory of ultracold atomic Fermi gases, Rev. Mod. Phys. 80, 1215.

Girardeau, M., 1960, Bosons and Fermions in One Dimension, J. Math. Phys. 1, 516.

Girardeau, M., H. Nguyen, and M. Olshanii, 2004, Effective interactions, FermiBose duality, and ground states of ultracold atomic vapors in tight de Broglie waveguides, Opt. Commun. 243, 3.

Gurarie, V. and L. Radzihovsky , 2007, Resonantly-paired fermionic superfluids, Ann. Phys. 322, 2.

Greiner, M., C. A. Regal, and D. S. Jin, 2003, Emergence of a Molecular Bose-Einstein Condensate from a Fermi Gas, Nature 426, 537 (2003).

Hadzibabic, Z. and J. Dalibard, 2011, Two-dimensional Bose fluids: An atomic physics perspective, Rivista Nuovo Cimento 34389.

Hadzibabic, Z. P. Kruger, M. Cheneau, B. Battelier, and J. Dalibard, 2006, BerezinskiiKosterlitzThouless crossover in a trapped atomic gas, Nature 4411118. 
He, L., H. Lü, G. Cao, H. Hu, and X.-J. Liu, 2015, Quantum fluctuations in the BCS-BEC crossover of two-dimensional Fermi gases, Phys. Rev. A 92023620.

Hohenberg, P.C., 1967, Existence of Long-Range Order in One and Two Dimensions, Phys. Rev. 158, 383.

Hu, H., X.-J. Liu, and P. Drummond, 2006, Equation of state of a superfluid Fermi gas in the BCS-BEC crossover, Europhys. Lett. 74, 574.

Hung, C.-L., X. Zhang, N. Gemelke, and C. Chin, 2011, Observation of scale invariance and universality in two-dimensional Bose gases, Nature 470, 236 .

Huang, K., 1987, Statistical Mechanics (Wiley, New York).

Kaku, M., 1993, Quantum Field Theory. A Modern Introduction (Oxford, Univ. Press), cap. 14.5.

Kapusta, J.I., 1993, Finite-Temperature Field Theory (Cambridge Univ. Press, Cambridge).

Kinoshita, T, T. Wenger, and D.S. Weiss, 2004, Observation of a onedimensional Tonks-Girardeau gas, Science 305, 1125.

Klimin, S.N., J. Tempere, and J.T. Devreese, 2012, Pseudogap and preformed pairs in the imbalanced Fermi gas in two dimensions New J. Phys. 14, 103044 .

Kosterlitz, J.M., and D.J. Thouless, 1973, Ordering, metastability and phase transitions in two-dimensional systems, J. Phys. C: Solid State Phys. 6, 1181.

Le Bellac, M., 1996, Thermal Field Theory (Cambridge Univ. Press, Cambridge).

Lee D.T., K. Huang and C.N. Yang, 1957, Eigenvalues and Eigenfunctions of a Bose System of Hard Spheres and Its Low-Temperature Properties, Phys. Rev. 106, 1135.

Leyronas X. and Combescot R., 2007, Superfluid Equation of State of Dilute Composite Bosons, Phys. Rev. Lett. 99, 170402.

Lee D.T. and C.N. Yang, 1957, Many-Body Problem in Quantum Mechanics and Quantum Statistical Mechanics, Phys. Rev. 105, 1119 
Leggett, A.J., 1980, Diatomic molecules and cooper pairs, Modern Trends in the Theory of Condensed Matter (Springer, Berlin), pp. 13-27.

Leggett, A.J., 2001, Bose-Einstein condensation in the alkali gases: Some fundamental concepts, Rev. Mod. Phys. 73, 307.

Leibbrandt, G., 1975, Introduction to the technique of dimensional regularization, Rev. Mod. Phys. 47, 849.

Liao, Y., A.S. C. Rittner, T. Paprotta, W. Li, G.B. Partridge, R.G. Hulet, S.K. Baur, and E.J. Mueller, 2010, Spin-imbalance in a one-dimensional Fermi gas, Nature 467, 567.

Lieb E.H. and W. Liniger, 1963, Exact Analysis of an Interacting Bose Gas. I. The General Solution and the Ground State, Phys. Rev. 130, 1605.

Luick N., 2014, Local probing of the Berezinskii-Kosterlitz-Thouless transition in a two-dimensional Bose gas, M.Sc. thesis, University of Hamburg, Supervisors: H. Moritz and L. Mathey.

Makhalov, V., K. Martiyanov, and A. Turlapov, 2014, Ground-State Pressure of Quasi-2D Fermi and Bose Gases, Phys. Rev. Lett. 112, 045301.

Moritz, H., T. Stoferle, K. Gunter, M. Kohl, and T. Esslinger, 2005, Confinement Induced Molecules in a 1D Fermi Gas, Phys. Rev. Lett. 94, 210401.

Manini, M. and L. Salasnich, 2005, Bulk and collective properties of a dilute Fermi gas in the BCS-BEC crossover, Phys. Rev. A 71, 033625.

Marini, M., F. Pistolesi, and G.C. Strinati, 1998, Evolution from BCS superconductivity to Bose condensation: analytic results for the crossover in three dimensions, Eur. Phys. J B 1, 151.

Mermin N.D. and H. Wagner, 1966, Absence of Ferromagnetism or Antiferromagnetism in One- or Two-Dimensional Isotropic Heisenberg Models, Phys. Rev. Lett. 17, 133.

Mora, C., and Y. Castin, 2003, Extension of Bogoliubov theory to quasicondensates, Phys. Rev. A 67, 053615.

Mora C., and Y. Castin, 2009, Ground state energy of the two-dimensional weakly interacting Bose gas: First correction beyond Bogoliubov theory, Phys. Rev. Lett. 102, 180404. 
Nagaosa, N., 1999, Quantum Field Theory in Condensed Matter Physics (Springer, Berlin).

Nascimbene, S., N. Navon, F. Chevy, and C. Salomon, 2010, The equation of state of ultracold Bose and Fermi gases: a few examples, New J. Phys. 12, 103026.

Navon, N., S. Nascimbene, F. Chevy, and C. Salomon, 2010, The equation of state of a low-temperature Fermi gas with tunable interactions, Science 328, 729 .

Nishida Y., and D.T. Son, 2006, Effective field theory of boson-fermion mixtures and bound fermion states on a vortex of boson superfluid, Phys. Rev. A 74, 013615.

Olshani, M., 1998, Atomic Scattering in the Presence of an External Confinement and a Gas of Impenetrable Bosons, Phys. Rev. Lett. 81, 938

Orso, G., 2007, Attractive Fermi Gases with Unequal Spin Populations in Highly Elongated Traps, Phys. Rev. Lett. 98, 070402

Papp, S.B., J. M. Pino, R. J. Wild, S. Ronen, C. E. Wieman, D. S. Jin, E. A. Cornell, 2008, Bragg spectroscopy of a strongly interacting ${ }^{85} \mathrm{Rb}$ Bose-Einstein condensate, Phys. Rev. Lett. 101, 135301.

Paredes B., A. Widera, V. Murg, O. Mandel, S. Folling, I. Cirac, G.V. Shlyapnikov, T.W. Hansch, and I. Bloch, 2004, Tonks-Girardeau gas of ultracold atoms in an optical lattice, Nature 429, 277.

Pauli, W. and F. Villars, 1949, On the Invariant Regularization in Relativistic Quantum Theory, Rev. Mod. Phys. 21, 434.

Petrov, D.S., C. Salomon, and G.V. Shlyapnikov, 2004, Weakly Bound Dimers of Fermionic Atoms, Phys. Rev. Lett. 93, 090404.

Pieri, P., and G. Strinati, 2000, Strong-coupling limit in the evolution from BCS superconductivity to Bose-Einstein condensation, Phys. Rev. B 61, 15370 .

Popov, V.N., 1972, On the theory of the superfluidity of two- and onedimensional Bose systems, Theor. Math. Phys. A 11, 565.

Pricoupenko, L., 2011, Isotopic contact forces in arbitrary representation: Heterogeneous few-body problems and low dimensions, Phys. Rev. A 83, 062711. 
Randeria, M., J-M. Duan, and L-Y. Shieh, 1989, Bound states, Cooper pairing, and Bose condensation in two dimensions, Phys. Rev. Lett. 62, 981.

Randeria, M., J-M. Duan, and L-Y. Shieh, 1990, Superconductivity in a two-dimensional Fermi gas: Evolution from Cooper pairing to Bose condensation, Phys. Rev. B 41, 327.

Salasnich, L., 2007, Condensate fraction of a two-dimensional attractive Fermi gas, Phys. Rev. A 76, 015601.

Salasnich, L., 2011, Condensate fraction in neutron matter, Phys. Rev. C 84, 067301.

Salasnich, L. and G. Bighin, 2015, Scattering length of composite bosons in the three-dimensional BCS-BEC crossover, Phys. Rev. A 91, 033610.

Salasnich, L., N. Manini, and A. Parola, 2005, Condensate fraction of a Fermi gas in the BCS-BEC crossover, Phys. Rev. A 72, 023621.

Salasnich, L. and F. Toigo, 2015, Composite bosons in the two-dimensional BCS-BEC crossover from Gaussian fluctuations, Phys. Rev. A 91, 011604(R).

Scalapino, D.J., 2012, A common thread: The pairing interaction for unconventional superconductors, Rev. Mod. Phys. 84, 1383.

Schakel, A.M.J., 1999, Bogoliubov's theory: a paradigm of quantum phase transitions, J. Phys. Studies 3, 337.

Schakel, A.M.J., 2008, Boulevard of Broken Symmetries (World Scientific, Singapore).

Schick, M., 1971, Two-Dimensional System of Hard-Core Bosons, Phys. Rev. A 3, 1067.

Shi, H. and A. Griffin, 1998, Finite-temperature excitations in a dilute Bosecondensed gas, Phys. Rep. 394, 1.

Stoof, H.T.C, K.B. Gubbels, and D.B.M. Dickerscheid, 2009, Ultracold Quantum Fields (Springer, Berlin).

Stringari, S., 2004, Collective oscillations of a trapped superfluid Fermi gas near a Feshbach resonance, Europhys. Lett., 65, 749. 
Tan, S., 2008, Large momentum part of a strongly correlated Fermi gas, Ann. Phys. (N.Y.), 323, 2971.

't Hooft, G. and M. Veltman, 1972, Regularization and Renormalization of Gauge Fields, Nucl. Phys. B 44, 189.

Yefsah, T., R. Desbuquois, L. Chomaz, K.J. Gunter, and J. Dalibard, 2011, Exploring the thermodynamics of a two-dimensional Bose gas, Phys. Rev. Lett. 107, 130401.

Wild, R.J., P. Makotyn, J.M. Pino, E.A. Cornell, and D.S. Jin, 2012, Measurements of Tan's contact in an atomic Bose-Einstein condensate, Phys. Rev. Lett. 108, 145305.

Zinner, N.T., B. Wunsch, D. Pekker, D.-W. Wang, 2012, BCS-BEC Crossover in Bilayers of Cold Fermionic Polar Molecules, Phys. Rev. A 85, 013603.

Zwerger, W. (Ed.), 2012, The BCS-BEC crossover and the unitary Fermi gas (Springer, Berlin). 